\title{
UNIVERSAL LAGRANGIAN BUNDLES
}

\author{
DANIELE SEPE
}

Department of Mathematics, University of Leicester, University Road, Leicester LE1 7RH, United Kingdom

\begin{abstract}
The obstruction to construct a Lagrangian bundle over a fixed integral affine manifold was constructed by Dazord and Delzant in [DD87] and shown to be given by 'twisted' cup products in [Sep11. This paper uses the topology of universal Lagrangian bundles, which classify Lagrangian bundles topologically (cf. Sep10b]), to reinterpret this obstruction as the vanishing of a differential on the second page of a Leray-Serre spectral sequence. Using this interpretation, it is shown that the obstruction of Dazord and Delzant depends on an important cohomological invariant of the integral affine structure on the base space, called the radiance obstruction, which was introduced by Goldman and Hirsch in GH84]. Some examples, related to non-degenerate singularities of completely integrable Hamiltonian systems, are discussed.
\end{abstract}

\section{Contents}

1. Introduction

2. Universal Lagrangian Bundles and Topological Invariants

3. Construction Problem

4. A Spectral Sequence

5. Relation to Integral Affine Geometry

6. Conclusion

References

\section{INTRODUCTION}

A fibre bundle $F \hookrightarrow(M, \omega) \rightarrow B$ is Lagrangian if $(M, \omega)$ is a $2 n$-dimensional symplectic manifold and the fibres are Lagrangian submanifolds, i.e. $\left.w\right|_{F}=0$ and $\operatorname{dim} F=n$. Throughout this paper, the fibres are assumed to be compact and connected. Lagrangian bundles arise naturally in many fields of mathematics, ranging from classical and quantum Hamiltonian mechanics to mirror symmetry, as the regular parts of the so-called Lagrangian fibrations. These admit singular fibres and are one of the geometric constructs used to study the topology, geometry and dynamics of completely integrable Hamiltonian systems (cf. Appendix D in [CB97]). Lagrangian bundles contain information about singularities of completely integrable Hamiltonian systems and are, therefore, central to the study of these dynamical systems. Under the above assumptions on the fibres, the Liouville-Mineur-Arnol'd theorem (cf. Section 49 in [Arn78]) implies that the fibres of a Lagrangian bundle are diffeomorphic to $n$-dimensional tori $\mathbb{T}^{n}$, and that a neighbourhood of a fibre is symplectomorphic to a neighbourhood of the zero section of the cotangent bundle $\left(\mathrm{T}^{*} B, \Omega_{\mathbb{T}^{n}}\right) \rightarrow \mathbb{T}^{n}$, where $\Omega_{\mathbb{T}^{n}}$ is the canonical symplectic form on $\mathrm{T}^{*} \mathbb{T}^{n}$. This theorem gives semi-global (i.e. in the neighbourhood of a fibre) topological and symplectic classifications of Lagrangian bundles. The global topological classification has been achieved by Duistermaat in [Dui80]; there are only two topological invariants, namely the topological monodromy and the Chern class, which is the obstruction to the existence of a section. Dazord and Delzant extended these notions to the broader class of isotropic bundles (whose fibres are not necessarily

E-mail address: dsepe@math.ist.utl.pt.

1991 Mathematics Subject Classification. 37J35, 53A15, 57R17, 70H06.

The author would like to thank José Figueroa-O'Farrill and Jarek Kedra for insightful comments on earlier versions of this article, and the anonymous referee for pointing out how to improve a previous version of the paper. 
of maximal dimension) in [DD87; moreover, they introduced a symplectic invariant, the Lagrangian class, which is the obstruction to the existence of a Lagrangian section.

Closely related to the classification problem is the question of constructing Lagrangian bundles over a fixed manifold $B$. The Liouville-Mineur-Arnol'd theorem, along with an observation due to Markus and Meyer in MM74 and Duistermaat Dui80, provides a necessary condition for a $\mathbb{T}^{n}$-bundle to be Lagrangian, namely that the fibres admit a smoothly varying affine structure, which is a $\mathrm{C}^{\infty}$-atlas $\mathcal{A}$ whose changes of coordinates are constant affine transformations of $\mathbb{R}^{n}$. Such bundles are called affine $\mathbb{R}^{n} / \mathbb{Z}^{n}$-bundles. The above condition is not sufficient (cf. [Bai01, Sep10b), as the total space does not necessarily admit an appropriate symplectic form. The existence of such a form on the total space of $\mathbb{T}^{n} \hookrightarrow(M, \omega) \rightarrow B$ implies that the base space is an integral affine manifold, i.e. an affine manifold whose atlas $\mathcal{A}$ has coordinate changes lying in

$$
\operatorname{Aff}_{\mathbb{Z}}\left(\mathbb{R}^{n}\right):=\mathrm{GL}(n ; \mathbb{Z}) \ltimes \mathbb{R}^{n} .
$$

This property restricts the topology of manifolds that arise as the base space of Lagrangian bundles: for instance, the only closed orientable (integral) affine surface is $\mathbb{T}^{2}$ (cf. Ben60, Mil58). The above observation, originally due to Duistermaat in Dui80, entwines the construction problem of Lagrangian bundles with the study of affine differential geometry, which was first introduced in the '50s and has since attracted much attention (e.g. AM55, FGH81, GH84, GH86, Mil58, Smi81).

Let $\mathbb{T}^{n} \hookrightarrow(M, \omega) \rightarrow B$ be a Lagrangian bundle, denote by $\chi_{*}: \pi_{1}(B ; b) \rightarrow \mathrm{GL}(n ; \mathbb{Z})$ its monodromy (cf. Definition 2.10) and let $\mathcal{A}$ be the induced integral affine atlas on $B$. Associated to $\mathcal{A}$ is a flat, torsion-free connection $\nabla$ on the tangent bundle TB (cf. AM55); denote by

$$
\mathfrak{l}: \pi_{1}(B ; b) \rightarrow \mathrm{GL}(n ; \mathbb{Z})
$$

its linear holonomy representation. Then

$$
\chi_{*}=\mathfrak{l}^{-T}
$$

where $-T$ denotes inverse transposed. Therefore, in order to study the construction problem for Lagrangian bundles over $B$, it is necessary to fix an integral affine structure $\mathcal{A}$ on $B$ and consider affine $\mathbb{R}^{n} / \mathbb{Z}^{n}$-bundles over $(B, \mathcal{A})$ whose monodromy satisfies equation (11). Such bundles are called almost Lagrangian (cf. Definition 3.12). The isomorphism class of these bundles depends on a cohomology class

$$
c \in \mathrm{H}^{2}\left(B ; \mathbb{Z}_{\chi_{*}}^{n}\right)
$$

i.e. the obstruction to the existence of a section. A result due to Dazord and Delzant in [DD87] shows that there is a homomorphism

$$
\mathcal{D}_{(B, \mathcal{A})}: \mathrm{H}^{2}\left(B ; \mathbb{Z}_{\chi_{*}}^{n}\right) \rightarrow \mathrm{H}^{3}(B ; \mathbb{R})
$$

whose kernel gives the subgroup of classes whose corresponding almost Lagrangian bundles are Lagrangian. This homomorphism can be computed explicitly and is obtained by taking a 'twisted' cup product on $B$, as shown in Sep11. However, the work of Sep11 leaves some natural questions regarding $\mathcal{D}_{(B, \mathcal{A})}$ unanswered, namely

- given that $\mathcal{D}_{(B, \mathcal{A})}$ measures, in some sense, the obstruction to constructing a suitable symplectic form on the total space of an almost Lagrangian bundle, can it be related to some differentials in the Leray-Serre spectral sequence of said bundle?

- what is the exact relation between $\mathcal{D}_{(B, \mathcal{A})}$ and the integral affine geometry of $(B, \mathcal{A})$ ?

- the twisted cup product of Definition 4 in Sep11 does not come from a pairing between $\mathrm{H}^{2}\left(B ; \mathbb{Z}_{\chi_{*}}^{n}\right)$ and some other cohomology group on $B$. Can $\mathcal{D}_{(B, \mathcal{A})}$ be understood as an 'honest' twisted cup product, i.e. for any $c \in \mathrm{H}^{2}\left(B ; \mathbb{Z}_{\chi_{*}}^{n}\right)$, is $\mathcal{D}_{(B, \mathcal{A})}(c)$ obtained by pairing $c$ with some suitable fixed cohomology class?

The main novelty of the paper is that it provides an answer to the above questions, thus providing a more natural, geometric description of $\mathcal{D}_{(B, \mathcal{A})}$. In particular, the main result of this paper shows that $\mathcal{D}_{(B, \mathcal{A})}$ is determined by an integral affine invariant of $(B, \mathcal{A})$, namely the radiance obstruction

$$
r_{(B, \mathcal{A})} \in \mathrm{H}_{2}^{1}\left(B ; \mathbb{R}_{\mathfrak{l}}^{n}\right) .
$$


This cohomology class has been defined by Goldman and Hirsch in GH84 (and implicitly by Smillie in Smi81]); it is the obstruction to homotoping the coordinate changes in $\mathcal{A}$ to take values in $\mathrm{GL}(n ; \mathbb{Z})$. In fact, the main result of the paper can be stated as follows.

Main Result. The obstruction for an almost Lagrangian bundle over an integral affine manifold $(B, \mathcal{A})$ with linear holonomy $\mathfrak{l}$ to be Lagrangian is given by

$$
\mathcal{D}_{(B, \mathcal{A})}(c)=c \cdot r_{(B, \mathcal{A})} \in \mathrm{H}^{3}(B ; \mathbb{R}),
$$

where $\cdot$ denotes the pairing in cohomology

$$
\mathrm{H}^{2}\left(B ; \mathbb{Z}_{\chi_{*}}^{n}\right) \otimes_{\mathbb{Z}} \mathrm{H}^{1}\left(B ; \mathbb{R}_{\mathfrak{l}}^{n}\right) \rightarrow \mathrm{H}^{3}(B ; \mathbb{R})
$$

obtained by combining cup product on $B$ with the duality between the coefficient systems.

Note that the above result implies Theorem 3 in [Sep11] and it also explains the geometric reason for why the 'twisted' cup product of Sep11] appears there in the first place (cf. Remark 5.11).

The Main Result is achieved by combining Theorems 4.6, 4.9 and 5.4 below. In particular, its proof can be broken down into the following three steps, each of which emphasises a new aspect of the Dazord-Delzant homomorphism.

Step 1. The Leray-Serre spectral sequence of universal Lagrangian bundles (cf. Sep10b]) is studied. These bundles classify, up to isomorphism, the topological type of affine $\mathbb{R}^{n} / \mathbb{Z}^{n}$-bundles and, in particular, of those which are Lagrangian. Each universal Lagrangian bundle has two topological invariants, namely its topological monodromy and the universal Chern class $c_{U}$. Using techniques from the work of Charlap and Vasquez CV66], Theorem 4.6 proves that some differential $\mathrm{d}^{(2)}$ on the $\mathrm{E}_{2}$-page of the Leray-Serre spectral sequence with $\mathbb{Z}$-coefficients of the universal Lagrangian bundle is given by taking cup products with the universal Chern class $c_{U}$. This is a non-trivial generalisation of the computation of the corresponding differential for the universal principal $\mathbb{T}^{n}$-bundle (cf. Lemma 4.4).

Step 2. Associated to an integral affine manifold $(B, \mathcal{A})$ is the symplectic reference bundle

$$
\mathbb{T}^{n} \hookrightarrow\left(\mathrm{T}^{*} B / P_{(B, \mathcal{A})}, \omega_{0}\right) \rightarrow B
$$

which admits a globally defined Lagrangian section and it induces the given integral affine atlas $\mathcal{A}$ on $B$. The symplectic form $\omega_{0}$ defines a cohomology class

$$
w_{0} \in \mathrm{H}^{1}\left(B ; \mathrm{H}^{1}\left(\mathbb{T}^{n} ; \mathbb{R}\right)_{\mathfrak{l}}\right),
$$

(cf. Lemma 4.7). Using Step 1, the obstruction for an almost Lagrangian bundle with Chern class $c$ to be Lagrangian is proved to be given by

$$
\mathrm{d}^{(2)}\left(w_{0}\right)=c \cdot w_{0}
$$

up to some isomorphisms (cf. Theorem 4.9). In light of the results in [DD87, this proves that the DazordDelzant homomorphism comes from the differential on the second page of the Leray-Serre spectral sequence of the underlying fibre bundle.

Step 3. The cohomology class $w_{0}$ is shown to be mapped to the radiance obstruction $r_{(B, \mathcal{A})}$ under an isomorphism induced by the symplectic form $\omega_{0}$ (cf. Theorem 5.4). Combining this result with Steps 1 and 2 above, obtain the Main Result, which expresses the Dazord-Delzant homomorphism as the pairing · with the radiance obstruction of $(B, \mathcal{A})$.

The structure of the paper is as follows. Section 2 constructs the universal Lagrangian bundles of Sep10b and the corresponding topological invariants, namely the monodromy and universal Chern class. These results only depend on the existence of a smoothly varying affine structure on the fibres of Lagrangian bundles. The importance of the integral affine geometry of the base space of a Lagrangian bundle is explained in Section 3, where the construction problem is posed and almost Lagrangian bundles are defined. Throughout this section, the connection with the sheaf theoretic point of view on the problem (cf. [DD87, Dui80]) is highlighted. Section 4 brings universal Lagrangian bundles into the problem of constructing Lagrangian bundles over an integral affine manifold $(B, \mathcal{A})$; Theorem 4.9 proves that the homomorphism $\mathcal{D}_{(B, \mathcal{A})}$ of 
Dazord and Delzant is given by taking twisted cup products with $w_{0}$, the cohomology class of the symplectic form of the symplectic reference bundle. This is obtained via a study of the Leray-Serre spectral sequence of universal Lagrangian bundles (cf. Theorem 4.6). Finally, Section 5 defines the radiance obstruction $r_{(B, \mathcal{A})}$ of an (integral) affine manifold $(B, \mathcal{A})$ and completes the proof of the Main Result stated above. Moreover, it exploits the connection between the symplectic topology of Lagrangian bundles and integral affine geometry in Theorem 5.5 and by considering some explicit examples which are related to singular Lagrangian bundles.

\section{Universal Lagrangian Bundles and Topological Invariants}

\subsection{Definition of universal Lagrangian bundles.}

Structure group. The Liouville-Mineur-Arnol'd theorem, along with a crucial observation due to Markus and Meyer MM74 and Duistermaat Dui80, can be used to prove the following theorem, stated below without proof, regarding the structure of Lagrangian bundles.

Theorem 2.1 (Markus and Meyer [MM74], Duistermaat [Dui80]). Let $F \hookrightarrow(M, \omega) \rightarrow B$ be a Lagrangian bundle. Then

i) for each $b \in B$, the fibre $F_{b}=\pi^{-1}(b)$ is diffeomorphic to $\mathbb{T}^{n}$;

ii) the structure group of the fibre bundle reduces to

$$
\operatorname{GL}(n ; \mathbb{Z}) \ltimes \mathbb{R}^{n} / \mathbb{Z}^{n}
$$

One of the consequences of Theorem 2.1 is that the fibres of a Lagrangian bundle are naturally affine manifolds.

Definition 2.1 (Affine manifolds). An affine structure on an $n$-dimensional manifold $B$ is a choice of atlas $\mathcal{A}=\left\{\left(U_{\alpha}, \phi_{\alpha}: U_{\alpha} \rightarrow \mathbb{R}^{n}\right)\right\}$ whose changes of coordinates

$$
\phi_{\beta} \circ \phi_{\alpha}^{-1}: \phi_{\alpha}\left(U_{\alpha} \cap U_{\beta}\right) \subset \mathbb{R}^{n} \rightarrow \phi_{\beta}\left(U_{\alpha} \cap U_{\beta}\right) \subset \mathbb{R}^{n}
$$

are constant on connected components, and are affine transformations of $\mathbb{R}^{n}$, i.e. they lie in the group

$$
\operatorname{Aff}\left(\mathbb{R}^{n}\right):=\operatorname{GL}(n ; \mathbb{R}) \ltimes \mathbb{R}^{n},
$$

where the action of $\mathrm{GL}(n ; \mathbb{R})$ on $\mathbb{R}^{n}$ is the standard one. An affine manifold is a pair $(B, \mathcal{A})$, where $B$ is a manifold and $\mathcal{A}$ is an affine structure on $B$.

In particular, the fibres of a Lagrangian bundle can be smoothly identified with the following affine manifold.

Example 2.2 (Standard affine structure on $\left.\mathbb{T}^{n}\right)$. Let $\Lambda \subset\left(\mathbb{R}^{n},+\right)$ be the standard cocompact lattice and let $\mathbf{e}^{1}, \ldots, \mathbf{e}^{n}$ denote the standard generators of $\Lambda$. Define a $\Lambda$-action on $\mathbb{R}^{n}$ by

$$
\mathbf{a} \cdot \mathbf{e}^{i}=\mathbf{a}+\mathbf{e}^{i},
$$

where $\mathbf{a}=\left(a^{1}, \ldots, a^{n}\right)$ denotes the standard (hence affine) coordinates on $\mathbb{R}^{n}$. The quotient $\mathbb{R}^{n} / \Lambda$ inherits an affine structure $\mathcal{A}$, since the above action is by affine diffeomorphisms of $\mathbb{R}^{n}$, i.e. diffeomorphisms which are affine in local affine coordinates. For notational ease, this affine manifold is henceforth denoted by $\mathbb{R}^{n} / \mathbb{Z}^{n}$.

Remark 2.3. Theorem 2.1 implies that the structure group of a Lagrangian bundle can be reduced to the group of affine diffeomorphisms of $\mathbb{R}^{n} / \mathbb{Z}^{n}$, denoted by $\operatorname{Aff}\left(\mathbb{R}^{n} / \mathbb{Z}^{n}\right)$.

Notation. The fibres of a Lagrangian bundle are henceforth denoted by $\mathbb{R}^{n} / \mathbb{Z}^{n}$ to emphasise the importance of their affine structure. Affine coordinates on $\mathbb{R}^{n} / \mathbb{Z}^{n}$ are denoted by $\boldsymbol{\theta}$.

Remark 2.4. The subgroup of $\operatorname{Aff}\left(\mathbb{R}^{n} / \mathbb{Z}^{n}\right)$ consisting of translations can be identified with the affine manifold $\mathbb{R}^{n} / \mathbb{Z}^{n}$ via the map that takes a translation $\mathcal{T}$ to $\mathcal{T} \boldsymbol{\theta}_{0}$, where $\boldsymbol{\theta}_{0} \in \mathbb{R}^{n} / \mathbb{Z}^{n}$ is any fixed point.

The existence of a smoothly varying affine structure on the fibres of a Lagrangian bundle $\mathbb{R}^{n} / \mathbb{Z}^{n} \hookrightarrow$ $(M, \omega) \rightarrow B$ implies the existence of an associated $\mathbb{Z}^{n}$-bundle $\mathbb{Z}^{n} \hookrightarrow P \rightarrow B$ whose fibres are smoothly identified with the lattice $\Lambda$ of Example 2.2

Definition 2.5 (Period lattice bundle [Dui80]). The covering space $\mathbb{Z}^{n} \hookrightarrow P \rightarrow B$ is called the period lattice bundle associated to $\mathbb{R}^{n} / \mathbb{Z}^{n} \hookrightarrow(M, \omega) \rightarrow B$. 
Definition of universal Lagrangian bundles. The topology of the group $\operatorname{Aff}\left(\mathbb{R}^{n} / \mathbb{Z}^{n}\right)$ can be used to construct universal Lagrangian bundles. Let 0 and 1 denote the trivial group with additive and multiplicative structures respectively. There is a short exact sequence of groups

$$
0 \longrightarrow \mathbb{R}^{n} / \mathbb{Z}^{n} \stackrel{\tau}{\longrightarrow} \operatorname{Aff}\left(\mathbb{R}^{n} / \mathbb{Z}^{n}\right) \stackrel{\text { Lin }}{\longrightarrow} \mathrm{GL}(n ; \mathbb{Z}) \longrightarrow 1,
$$

where the homomorphisms $\tau, p$ are defined by

$$
\begin{aligned}
\tau: \mathbb{R}^{n} / \mathbb{Z}^{n} & \rightarrow \operatorname{Aff}\left(\mathbb{R}^{n} / \mathbb{Z}^{n}\right) \\
\boldsymbol{\theta} & \mapsto(I, \boldsymbol{\theta}) \\
\operatorname{Lin}: \operatorname{Aff}\left(\mathbb{R}^{n} / \mathbb{Z}^{n}\right) & \rightarrow \operatorname{GL}(n ; \mathbb{Z}) \\
(A, \boldsymbol{\theta}) & \mapsto A,
\end{aligned}
$$

and $I$ denotes the identity in $\mathrm{GL}(n ; \mathbb{Z})$. The sequence of equation (2) admits a splitting

$$
\begin{aligned}
\sigma: \operatorname{GL}(n ; \mathbb{Z}) & \rightarrow \operatorname{Aff}\left(\mathbb{R}^{n} / \mathbb{Z}^{n}\right) \\
A & \mapsto(A, \mathbf{0}),
\end{aligned}
$$

where $\mathbf{0}$ denotes the identity in the group $\mathbb{R}^{n} / \mathbb{Z}^{n}$. The injections $\sigma, \tau$ defined above give rise to interesting bundles via the following well-known theorem, stated below without proof (cf. Section 5 in [Hus93]).

Theorem 2.2. Let $G, H$ be topological groups and let $\iota: H \hookrightarrow G$ be a monomorphism. There exists a bundle

$$
G / H \hookrightarrow \mathrm{B} H \rightarrow \mathrm{B} G,
$$

where $\mathrm{B} G, \mathrm{~B} H$ are the classifying spaces for $G$ and $H$ respectively. If, in addition, $\iota(H) \triangleleft G$, then the above bundle is a $G / H$-principal bundle.

Applying Theorem 2.2 to the inclusion $\tau: \mathbb{R}^{n} / \mathbb{Z}^{n} \rightarrow \operatorname{Aff}\left(\mathbb{R}^{n} / \mathbb{Z}^{n}\right)$ and to the splitting $\sigma: \mathrm{GL}(n ; \mathbb{Z}) \rightarrow$ $\operatorname{Aff}\left(\mathbb{R}^{n} / \mathbb{Z}^{n}\right)$ defined above, obtain two bundles

$$
\begin{aligned}
& \mathrm{GL}(n ; \mathbb{Z}) \longrightarrow \mathrm{B}\left(\mathbb{R}^{n} / \mathbb{Z}^{n}\right) \stackrel{\tau}{\longrightarrow} \mathrm{BAff}_{\mathbb{Z}}\left(\mathbb{R}^{n} / \mathbb{Z}^{n}\right) \\
& \mathbb{R}^{n} / \mathbb{Z}^{n \smile} \longrightarrow \mathrm{BGL}(n ; \mathbb{Z}) \stackrel{\sigma}{\longrightarrow} \mathrm{BAff}_{\mathbb{Z}}\left(\mathbb{R}^{n} / \mathbb{Z}^{n}\right)
\end{aligned}
$$

Remark 2.6. Note that since $\sigma(\operatorname{GL}(n ; \mathbb{Z}))$ is not a normal subgroup of $\operatorname{Aff}\left(\mathbb{R}^{n} / \mathbb{Z}^{n}\right)$, the fibres of the bundle of equation (3b) are not naturally endowed with the structure of a group.

Definition 2.7 (Universal Lagrangian bundles [Sep10b]). For each $n$, the bundle of equation (3b) is called the universal Lagrangian bundle of dimension $n$.

Notation. Throughout this paper, $n$ is fixed, unless otherwise stated, and the universal Lagrangian bundle of dimension $n$ is referred to simply as the universal Lagrangian bundle.

Remark 2.8. The fibres of a universal Lagrangian bundle are endowed with an affine structure which makes them affinely diffeomorphic to $\mathbb{R}^{n} / \mathbb{Z}^{n}$. This is because the subgroup $\tau\left(\mathbb{R}^{n} / \mathbb{Z}^{n}\right)$ of Aff $\left(\mathbb{R}^{n} / \mathbb{Z}^{n}\right)$ can be identified with the affine manifold $\mathbb{R}^{n} / \mathbb{Z}^{n}$ (cf. Remark 2.4).

The following lemma states what the homotopy groups of BAff $\left(\mathbb{R}^{n} / \mathbb{Z}^{n}\right)$ are; its proof is omitted as it can be found in Sep10b].

Lemma 2.3 (Homotopy groups of BAff $\left(\mathbb{R}^{n} / \mathbb{Z}^{n}\right)$ Sep10b). The homotopy groups of BAff $\left(\mathbb{R}^{n} / \mathbb{Z}^{n}\right)$ are given by

$$
\pi_{i}\left(\operatorname{BAff}\left(\mathbb{R}^{n} / \mathbb{Z}^{n}\right)\right)= \begin{cases}\mathrm{GL}(n ; \mathbb{Z}) & \text { if } i=1, \\ \mathbb{Z}^{n} & \text { if } i=2, \\ 0 & \text { otherwise. }\end{cases}
$$


Universality. Fix a Lagrangian bundle $\mathbb{R}^{n} / \mathbb{Z}^{n} \hookrightarrow(M, \omega) \rightarrow B$. In light of Theorem 2.1] it is classified topologically by the homotopy class of a map

$$
\chi: B \rightarrow \operatorname{BAff}\left(\mathbb{R}^{n} / \mathbb{Z}^{n}\right),
$$

which is the classifying map of the associated principal $\operatorname{Aff}\left(\mathbb{R}^{n} / \mathbb{Z}^{n}\right)$-bundle. The following theorem shows that the bundles of Definition 2.7 are universal in the sense that $\mathbb{R}^{n} / \mathbb{Z}^{n} \hookrightarrow(M, \omega) \rightarrow B$ is isomorphic to the pull-back of the universal Lagrangian bundle along $\chi$. The result below is presented without proof as it follows from a simple observation for general semidirect products.

Theorem 2.4 (Universality). Let $\mathbb{R}^{n} / \mathbb{Z}^{n} \hookrightarrow(M, \omega) \rightarrow B$ be a Lagrangian bundle and let $\chi: B \rightarrow$ $\operatorname{BAff}\left(\mathbb{R}^{n} / \mathbb{Z}^{n}\right)$ denote its classifying map. Then $\mathbb{R}^{n} / \mathbb{Z}^{n} \hookrightarrow(M, \omega) \rightarrow B$ is isomorphic to the pull-back of the universal Lagrangian bundle along $\chi$

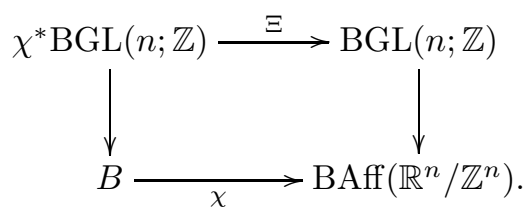

Remark 2.9. As observed in the literature (e.g. BS92, Dui80, Luk08), the fibres of a Lagrangian bundle are not naturally equipped with a group structure. This can be proved directly using Theorem 2.4 and Remark 2.6.

2.2. Topological invariants. In light of Theorem 2.4 the topological invariants of Lagrangian bundles are pull-backs of universal invariants, which characterise the universal Lagrangian bundle.

Monodromy. Fix a Lagrangian bundle $\mathbb{R}^{n} / \mathbb{Z}^{n} \hookrightarrow(M, \omega) \rightarrow B$ with classifying map $\chi: B \rightarrow \operatorname{BAff}\left(\mathbb{R}^{n} / \mathbb{Z}^{n}\right)$.

Definition 2.10 (Monodromy [Sep10b]). Let $b_{0} \in B$ be a basepoint. The monodromy of $\mathbb{R}^{n} / \mathbb{Z}^{n} \hookrightarrow$ $(M, \omega) \rightarrow B$ is defined to be the homomorphism

$$
\chi_{*}: \pi_{1}\left(B ; b_{0}\right) \rightarrow \pi_{1}\left(\operatorname{BAff}\left(\mathbb{R}^{n} / \mathbb{Z}^{n}\right) ; \chi\left(b_{0}\right)\right) \cong \operatorname{GL}(n ; \mathbb{Z}) .
$$

\section{Remark 2.11.}

i) There is a notion of universal monodromy, which arises from considering the classifying map of the universal Lagrangian bundle

$$
\mathbb{R}^{n} / \mathbb{Z}^{n} \hookrightarrow \operatorname{BAff}\left(\mathbb{R}^{n} / \mathbb{Z}^{n}\right) \rightarrow \operatorname{BGL}(n ; \mathbb{Z}) .
$$

This map is (up to homotopy) just the identity id : $\operatorname{BAff}\left(\mathbb{R}^{n} / \mathbb{Z}^{n}\right) \rightarrow \operatorname{BAff}\left(\mathbb{R}^{n} / \mathbb{Z}^{n}\right)$;

ii) The choice of base point $b_{0} \in B$ may affect the image of the homomorphism $\chi_{*}$, without, however, changing its conjugacy class in $\mathrm{GL}(n ; \mathbb{Z})$. The free monodromy of a Lagrangian bundle $\mathbb{R}^{n} / \mathbb{Z}^{n} \hookrightarrow$ $(M, \omega) \rightarrow B$ is defined to be the conjugacy class $\left[\chi_{*}\right]$ of the image of the monodromy and is therefore independent of the choice of base point $b \in B$;

iii) The bundle

$$
\mathrm{GL}(n ; \mathbb{Z}) \longleftrightarrow \mathrm{B}\left(\mathbb{R}^{n} / \mathbb{Z}^{n}\right) \stackrel{\tau}{\longrightarrow} \mathrm{BAff}_{\mathbb{Z}}\left(\mathbb{R}^{n} / \mathbb{Z}^{n}\right)
$$

of equation (3a) is the universal covering of $\operatorname{BAff}\left(\mathbb{R}^{n} / \mathbb{Z}^{n}\right)$. Thus if the (free) monodromy $\chi_{*}$ of a Lagrangian bundle $\mathbb{R}^{n} / \mathbb{Z}^{n} \hookrightarrow(M, \omega) \rightarrow B$ is trivial, the classifying map $\chi$ admits a lift $\tilde{\chi}: B \rightarrow$ $\mathrm{B}\left(\mathbb{R}^{n} / \mathbb{Z}^{n}\right)$. This implies that the structure group of the Lagrangian bundle can be reduced to the group $\mathbb{R}^{n} / \mathbb{Z}^{n}$ (cf. Theorem 5.1 in [Hus93]).

The monodromy of a Lagrangian bundle $\mathbb{R}^{n} / \mathbb{Z}^{n} \hookrightarrow(M, \omega) \rightarrow B$ was originally defined to be the homotopy class of the classifying map of the associated period lattice bundle $\mathbb{Z}^{n} \hookrightarrow P \rightarrow B$ (cf. [Dui80]). This can be seen using universal Lagrangian bundles.

Definition 2.12 (Universal period lattice bundles). The universal period lattice bundle associated to the universal Lagrangian bundle

$$
\mathbb{R}^{n} / \mathbb{Z}^{n} \hookrightarrow \operatorname{BAff}\left(\mathbb{R}^{n} / \mathbb{Z}^{n}\right) \rightarrow \operatorname{BGL}(n ; \mathbb{Z})
$$

is the induced system of local coefficients with fibre $\mathrm{H}_{1}\left(\mathbb{R}^{n} / \mathbb{Z}^{n} ; \mathbb{Z}\right)$, denoted by

$$
\mathrm{H}_{1}\left(\mathbb{R}^{n} / \mathbb{Z}^{n} ; \mathbb{Z}\right) \hookrightarrow P_{n} \rightarrow \mathrm{BAff}_{\mathbb{Z}}\left(\mathbb{R}^{n} / \mathbb{Z}^{n}\right) .
$$


The pull-back $\chi^{*} P_{n} \rightarrow B$ is isomorphic to the system of local coefficients obtained by replacing each fibre $\mathbb{R}^{n} / \mathbb{Z}^{n}$ of $\chi^{*} \operatorname{BGL}(n ; \mathbb{Z}) \rightarrow B$ with $\mathrm{H}_{1}\left(\mathbb{R}^{n} / \mathbb{Z}^{n} ; \mathbb{Z}\right)$. By Theorem $2.4 \chi^{*} \operatorname{BGL}(n ; \mathbb{Z}) \rightarrow B$ is isomorphic to the Lagrangian bundle $\mathbb{R}^{n} / \mathbb{Z}^{n} \hookrightarrow(M, \omega) \rightarrow B$ whose classifying map is given by $\chi$. The following lemma, stated below without proof, shows that $\chi^{*} P_{n} \rightarrow B$ is isomorphic to the period lattice bundle $P \rightarrow B$ associated to $\mathbb{R}^{n} / \mathbb{Z}^{n} \hookrightarrow(M, \omega) \rightarrow B$. Its proof follows from ideas in Dui80].

Lemma 2.5. Let $P \rightarrow B$ denote the period lattice bundle associated to the Lagrangian bundle $\mathbb{R}^{n} / \mathbb{Z}^{n} \hookrightarrow$ $(M, \omega) \rightarrow B$ (with classifying map $\chi$ ) as in Definition 2.5. Then $\chi^{*} P_{n} \rightarrow B$ is isomorphic to $P \rightarrow B$.

The (free) monodromy $\chi_{*}$ of the Lagrangian bundle $\mathbb{R}^{n} / \mathbb{Z}^{n} \hookrightarrow(M, \omega) \rightarrow B$ determines the homotopy class of the classifying map of $\chi^{*} P_{n} \rightarrow B$, which is isomorphic to the period lattice bundle $P \rightarrow B$ by Lemma 2.5. This proves that the monodromy of Definition 2.10 defined above is equivalent to the notion given in Dui80.

Chern class. Free monodromy is not the only topological invariant of a Lagrangian bundle. This can be seen by considering the case when the (free) monodromy vanishes and the topological classification reduces to that of principal $\mathbb{R}^{n} / \mathbb{Z}^{n}$-bundles. In this case, there is only one other topological invariant associated to the given fibre bundle, namely the obstruction to the existence of a section. In what follows, the corresponding obstruction for any Lagrangian bundle is defined using universal Lagrangian bundles.

The approach taken below is obstruction theoretic, which is suitable since all spaces involved are homotopic to CW complexes. The idea is to find the obstruction to the existence of a section of the universal Lagrangian bundle. A section of this bundle is a lift of the identity map id : $\operatorname{BAff}\left(\mathbb{R}^{n} / \mathbb{Z}^{n}\right) \rightarrow \operatorname{BAff}\left(\mathbb{R}^{n} / \mathbb{Z}^{n}\right)$, i.e. a map (denoted by the dotted arrow) which makes the following diagram commute

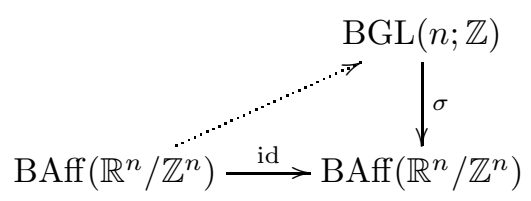

As the total space and the fibres are path-connected, a section can be defined on the 1-skeleton of BAff $\left(\mathbb{R}^{n} / \mathbb{Z}^{n}\right)$. The problem of extending this section to the 2 -skeleton can be tackled cell by cell; however, there needs to be extra care as BAff $\left(\mathbb{R}^{n} / \mathbb{Z}^{n}\right)$ is not simply connected. Set $\varpi=\pi_{1}\left(\mathrm{BAff}\left(\mathbb{R}^{n} / \mathbb{Z}^{n}\right)\right)$, fix a CW decomposition of $\operatorname{BAff}\left(\mathbb{R}^{n} / \mathbb{Z}^{n}\right)$ and a $\varpi$-equivariant CW decomposition of its universal cover BAff( $\left.\widetilde{\mathbb{R}^{n}} / \mathbb{Z}^{n}\right)$. Using standard techniques in obstruction theory (cf. Theorem 7.37 in [DK01, Theorem 5.5 in [Whi78]), it can be shown that the obstruction to the existence of an extension to the 2-skeleton is a cocycle in

$$
\operatorname{Hom}_{\mathbb{Z}[\varpi]}\left(\mathrm{C}_{2}\left(\mathrm{BAff}\left(\widetilde{\mathbb{R}^{n}} / \mathbb{Z}^{n}\right)\right) ; \mathbb{Z}^{n}\right)
$$

where $\mathrm{C}_{2}\left(\mathrm{BAff}\left(\widetilde{\mathbb{R}^{n}} / \mathbb{Z}^{n}\right)\right)$ and $\mathbb{Z}^{n}$ are naturally $\mathbb{Z}[\varpi]$-modules: the former because of the choice of $\varpi$ equivariant $\mathrm{CW}$ decomposition of $\mathrm{BAff( \mathbb {R } ^ { n } / \mathbb { Z } ^ { n } )}$ and the latter via the topological monodromy representation of the topological universal Lagrangian bundle.

Definition 2.13 (Universal Chern class). The cohomology class of the above cocycle

$$
c_{U} \in \mathrm{H}^{2}\left(\operatorname{BAff}\left(\mathbb{R}^{n} / \mathbb{Z}^{n}\right) ; \mathbb{Z}_{\mathrm{id}_{*}}^{n}\right),
$$

is called the universal Chern class.

The importance of the universal Chern class $c_{U}$ is highlighted in Section 4 where it is used to study the Leray-Serre spectral sequence associated to the universal Lagrangian bundle.

Definition 2.14. Let $\mathbb{R}^{n} / \mathbb{Z}^{n} \hookrightarrow(M, \omega) \rightarrow B$ be a Lagrangian bundle with classifying map $\chi: B \rightarrow$ BAff $\left(\mathbb{R}^{n} / \mathbb{Z}^{n}\right)$. Its Chern class is defined to be the pull-back

$$
\chi^{*} c_{U} \in \mathrm{H}^{2}\left(B ; \mathbb{Z}_{\chi_{*}}^{n}\right) .
$$

\section{Remark 2.15.}


i) A priori, the cohomology class $\chi^{*} c_{U}$ is only the primary obstruction to the existence of a section for $\mathbb{R}^{n} / \mathbb{Z}^{n} \hookrightarrow(M, \omega) \rightarrow B$, i.e. the vanishing of $\chi^{*} c_{U}$ may not mean that there exists a section $s: B \rightarrow M$. However, if $\chi^{*} c_{U}=0$, there exists a section defined on the 2 -skeleton of $B$; it then follows from standard arguments in obstruction theory that this section can be extended to $B$. Thus $\chi^{*} c_{U}$ is the only obstruction to the existence of a section;

ii) In Sep10b the Chern class of a Lagrangian bundle is defined to be the obstruction to the existence of a lift

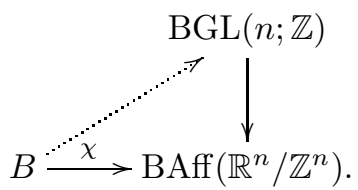

This obstruction coincides with the obstruction to the existence of a section $s: B \rightarrow \chi^{*} \operatorname{BGL}(n ; \mathbb{Z})$. Since $\mathbb{R}^{n} / \mathbb{Z}^{n} \hookrightarrow(M, \omega) \rightarrow B$ is isomorphic to the pull-back of the universal Lagrangian bundle along $\chi$, the latter is just $\chi^{*} c_{U}$ and the above definition coincides with that of Sep10b.

Sharpness. The two topological invariants defined above completely determine the topological type of a Lagrangian bundle, as mentioned in Bat88, DD87, Dui80, Luk08, Ngo03, Zun03. This can also be seen using arguments in equivariant obstruction theory which are akin to those used to define the universal Chern class. Thus the following sharpness theorem is only stated.

Theorem 2.6 (Sharpness [DD87, Dui80, Luk08, Sep10b). Two Lagrangian bundles

$$
\mathbb{R}^{n} / \mathbb{Z}^{n} \hookrightarrow(M, \omega) \rightarrow B \quad, \quad \mathbb{R}^{n} / \mathbb{Z}^{n} \hookrightarrow\left(M^{\prime}, \omega^{\prime}\right) \rightarrow B
$$

are isomorphic (as fibre bundles) if and only if their free monodromies and Chern classes coincide.

\section{Construction Problem}

3.1. Relation between integral affine geometry and Lagrangian bundles. he classification theory developed in Section 2 does not suffice to tackle the problem of constructing Lagrangian bundles over a fixed base space $B$. This is because the above classification is merely topological and not symplectic. In fact, the (free) monodromy and the Chern class are not unique to Lagrangian bundles, as illustrated below.

Definition 3.1 (Affine $\mathbb{R}^{n} / \mathbb{Z}^{n}$-bundles [Bai01]). A locally trivial $\mathbb{T}^{n}$-bundle is called affine if its structure group reduces to $\mathrm{Aff}\left(\mathbb{R}^{n} / \mathbb{Z}^{n}\right)$.

Remark 3.2. The fibres of an affine $\mathbb{R}^{n} / \mathbb{Z}^{n}$-bundle can be smoothly identified with the affine manifold $\mathbb{R}^{n} / \mathbb{Z}^{n}$. The topological classification of affine $\mathbb{R}^{n} / \mathbb{Z}^{n}$-bundles can be carried out as in Section 2 above, since their structure group reduces to $\operatorname{Aff}\left(\mathbb{R}^{n} / \mathbb{Z}^{n}\right)$; in particular, Theorem 2.6 applies to this more general class.

Not all locally trivial $\mathbb{T}^{n}$-bundles are affine $\mathbb{R}^{n} / \mathbb{Z}^{n}$-bundles as illustrated in an example due to Baier in Bai01. Moreover, it can be shown that not all affine $\mathbb{R}^{n} / \mathbb{Z}^{n}$-bundles are Lagrangian by considering the case of principal $\mathbb{R}^{n} / \mathbb{Z}^{n}$-bundles over $S^{n}$ (cf. SSep10b]). What distinguishes affine $\mathbb{R}^{n} / \mathbb{Z}^{n}$ from Lagrangian bundles is the existence of a suitable symplectic form on the total space; this object imposes severe restrictions on the topology of the base space, as illustrated in the next section.

Action-angle coordinates and integral affine geometry. One of the crucial consequences of the LiouvilleMineur-Arnol'd theorem is the existence of canonical coordinates in a neighbourhood of a fibre of a Lagrangian bundle. These are known as action-angle coordinates and play a very important role in Hamiltonian mechanics. The following theorem illustrates the existence of such coordinates and investigates how they change; it is stated below without proof as it is well-known.

Theorem 3.1 (Existence of local action-angle coordinates, Section 49 in Arn78, Dui80]). Let $F \hookrightarrow$ $(M, \omega) \rightarrow B$ be a Lagrangian bundle. There exists an open cover $\mathcal{U}=\left\{U_{\alpha}\right\}$ of $B$ and coordinate charts $\phi_{\alpha}: U_{\alpha} \rightarrow \mathbb{R}^{n}$ inducing symplectic trivialisations

$$
\Upsilon_{\alpha}:\left(\mathrm{T}^{*} U_{\alpha} / P_{\alpha}, \omega_{0, \alpha}\right) \rightarrow\left(\pi^{-1}\left(U_{\alpha}\right), \omega_{\alpha}\right),
$$


where $P_{\alpha} \subset\left(\mathrm{T}^{*} U_{\alpha}, \Omega_{\alpha}\right)$ is the Lagrangian submanifold

$$
P_{\alpha}=\left\{\left(\mathbf{a}_{\alpha}, \mathbf{p}_{\alpha}\right) \in \mathrm{T}^{*} U_{\alpha}: \mathbf{p}_{\alpha} \in \mathbb{Z}\left\langle\mathrm{d} a_{\alpha}^{1}, \ldots, \mathrm{d} a_{\alpha}^{n}\right\rangle\right\},
$$

$a_{\alpha}^{1}, \ldots, a_{\alpha}^{n}$ are local coordinates on $U_{\alpha}$ induced by $\phi_{\alpha}$, and $\left(\mathbf{a}_{\alpha}, \mathbf{p}_{\alpha}\right)$ are canonical coordinates on $\mathrm{T}^{*} U_{\alpha}$. The local coordinates $\left(\mathbf{a}_{\alpha}, \boldsymbol{\theta}_{\alpha}\right)$ induced by $\Upsilon_{\alpha}$ are called action-angle coordinates and satisfy

$$
\Upsilon_{\alpha}^{*} \omega_{\alpha}=\omega_{0, \alpha}=\sum_{i=1}^{n} \mathrm{~d} a_{\alpha}^{i} \wedge \mathrm{d} \theta_{\alpha}^{i}
$$

For the purposes of this work, the most important consequence of Theorem 3.1 is that the base space $B$ of a Lagrangian bundle inherits the structure of an integral affine manifold.

Definition 3.3 (Integral affine manifolds). An $n$-dimensional affine manifold $(B, \mathcal{A})$ is said to be integral if the coordinate changes in its affine structure $\mathcal{A}$ are integral affine maps of $\mathbb{R}^{n}$, i.e. elements of the group

$$
\operatorname{Aff}_{\mathbb{Z}}\left(\mathbb{R}^{n}\right):=\operatorname{GL}(n ; \mathbb{Z}) \ltimes \mathbb{R}^{n} .
$$

The following lemma shows that the coordinate neighbourhoods $\phi_{\alpha}$ of Theorem 3.1 give rise to an integral affine structure on $B$ and that, conversely, all integral affine manifolds arise as the base space of some Lagrangian bundle (for a proof, see Sep10b]).

Lemma 3.2 ([Dui80, Luk08, Sep10b]). A manifold B is the base space of a Lagrangian bundle if and only if it is an integral affine manifold.

Remark 3.4. Associated to an integral affine manifold $(B, \mathcal{A})$ is a Lagrangian submanifold $P_{(B, \mathcal{A})} \subset$ $\left(\mathrm{T}^{*} B, \Omega\right)$, locally given by

$$
P_{\alpha}:=\left\{\left(\mathbf{a}_{\alpha}, \mathbf{p}_{\alpha}\right) \in \mathrm{T}^{*} U_{\alpha}: \mathbf{p}_{\alpha} \in \mathbb{Z}\left\langle\mathrm{d} a_{\alpha}^{1}, \ldots, \mathrm{d} a_{\alpha}^{n}\right\rangle\right\},
$$

where $U_{\alpha} \subset B$ is a coordinate neighbourhood with integral affine coordinates $\mathbf{a}_{\alpha}$, and $\mathbf{p}_{\alpha}$ denote canonical coordinates on the fibres of $\mathrm{T}^{*} U_{\alpha}$. The canonical symplectic form $\Omega$ on $\mathrm{T}^{*} B$ descends to a symplectic form $\omega_{0}$ on the quotient $\mathrm{T}^{*} B / P_{(B, \mathcal{A})}$; thus,

$$
\left(\mathrm{T}^{*} B / P, \omega_{0}\right) \rightarrow B
$$

is a Lagrangian bundle with a globally defined Lagrangian section, namely the zero section.

Note that the construction of Remark 3.4 only depends on the integral affine structure on $B$ and, thus, the following are well-defined.

Definition 3.5 (Topological reference bundle Sep10a). Let $(B, \mathcal{A})$ be an integral affine manifold. The isomorphism class of the bundle

$$
\mathbb{R}^{n} / \mathbb{Z}^{n} \hookrightarrow\left(\mathrm{T}^{*} B / P_{(B, \mathcal{A})}, \omega_{0}\right) \rightarrow B
$$

as an affine $\mathbb{R}^{n} / \mathbb{Z}^{n}$-bundle is called the topological reference bundle for $(B, \mathcal{A})$.

Definition 3.6 (Symplectic reference bundle Sep10b, Zun03]). The bundle

$$
\mathbb{R}^{n} / \mathbb{Z}^{n} \hookrightarrow\left(\mathrm{T}^{*} B / P_{(B, \mathcal{A})}, \omega_{0}\right) \rightarrow B
$$

is the symplectic reference bundle for the integral affine manifold $(B, \mathcal{A})$.

Remark 3.7 (Symplectic reference bundle). It is important to notice that the symplectic reference bundle is equipped with a distinguished Lagrangian section. Any other Lagrangian bundle which admits a Lagrangian section is fibrewise symplectomorphic to the symplectic reference bundle, i.e. there exists a fibre bundle isomorphism which is also a symplectomorphism of the total spaces. Such a symplectomorphism is sometimes referred to as a polarisation in the literature, e.g. Mis96.

The submanifold $P_{(B, \mathcal{A})} \subset \mathrm{T}^{*} B$ constructed above plays an important role in building Lagrangian bundles.

Definition 3.8 (Period lattice bundle of an integral affine manifold). Let $(B, \mathcal{A})$ be an integral affine manifold. The Lagrangian submanifold $P_{(B, \mathcal{A})} \subset\left(\mathrm{T}^{*} B, \Omega\right)$ constructed in Remark 3.4 is called the period lattice bundle associated to $(B, \mathcal{A})$. 
Let $\mathbb{R}^{n} / \mathbb{Z}^{n} \hookrightarrow(M, \omega) \rightarrow B$ be a Lagrangian bundle. Theorem 2.1 and Lemma 3.2 show that $B$ can be covered by integral affine coordinate neighbourhoods $U_{\alpha}$ over which the bundle is trivial. Let $\left(\mathbf{a}_{\alpha}, \boldsymbol{\theta}_{\alpha}\right)$ be action-angle coordinates on $\pi^{-1}\left(U_{\alpha}\right)$, so that the restriction of $\omega$ to $\pi^{-1}\left(U_{\alpha}\right)$, denoted by $\omega_{\alpha}$, is

$$
\sum_{i=1}^{n} \mathrm{~d} a_{\alpha}^{i} \wedge \mathrm{d} \theta_{\alpha}^{i} .
$$

The transition functions $\varphi_{\beta \alpha}$ with respect to the above choice of local trivialisations are given by (cf. [Zun03])

$$
\varphi_{\beta \alpha}\left(\mathbf{a}_{\alpha}, \boldsymbol{\theta}_{\alpha}\right)=\left(A_{\beta \alpha} \mathbf{a}_{\alpha}+\mathbf{d}_{\beta \alpha}, A_{\beta \alpha}^{-T} \boldsymbol{\theta}_{\alpha}+\mathbf{g}_{\beta \alpha}\left(\mathbf{a}_{\alpha}\right)\right),
$$

where $\left(A_{\beta \alpha}, \mathbf{d}_{\beta \alpha}\right) \in \operatorname{Aff}_{\mathbb{Z}}\left(\mathbb{R}^{n}\right)$ is a change of integral affine coordinates, and $\mathbf{g}_{\beta \alpha}: U_{\alpha} \cap U_{\beta} \rightarrow \mathbb{R}^{n} / \mathbb{Z}^{n}$ is a smooth map which is constrained by

$$
\varphi_{\beta \alpha}^{*} \omega_{\beta}=\omega_{\alpha},
$$

i.e. the local symplectic forms patch together to yield $\omega$ on $M$.

Monodromy and integral affine geometry. Let $\mathbb{R}^{n} / \mathbb{Z}^{n} \hookrightarrow(M, \omega) \rightarrow B$ be a Lagrangian bundle. The induced integral affine structure $\mathcal{A}$ on the base space can be understood in terms of a flat, torsion-free connection $\nabla$ on $\mathrm{T} B$ whose holonomy takes values in $\mathrm{Aff}_{\mathbb{Z}}\left(\mathbb{R}^{n}\right)$ (cf. $\mathrm{AM55}$ ).

Definition 3.9 (Affine and linear holonomy [AM55, GH84]). The holonomy of $\nabla$, denoted by

$$
\mathfrak{a}: \pi_{1}(B ; b) \rightarrow \operatorname{Aff}_{\mathbb{Z}}\left(\mathbb{R}^{n}\right),
$$

is called the affine holonomy of the integral affine manifold $(B, \mathcal{A})$. Composing $\mathfrak{a}$ with the projection

$$
\operatorname{Lin}: \operatorname{Aff}_{\mathbb{Z}}\left(\mathbb{R}^{n}\right) \rightarrow \operatorname{GL}(n ; \mathbb{Z}),
$$

obtain the linear holonomy $\mathfrak{l}: \pi_{1}(B ; b) \rightarrow \mathrm{GL}(n ; \mathbb{Z})$.

Remark 3.10. The affine holonomy is only well-defined up to an explicit choice of affine structure and up to a choice of basepoint. Throughout this work, whenever an affine/linear holonomy homomorphism is considered, it is understood that the basepoint and the explicit integral affine structure are fixed.

The linear monodromy $\mathfrak{l}$ of $(B, \mathcal{A})$ is related to the monodromy $\chi_{*}$ of the Lagrangian bundle $\mathbb{R}^{n} / \mathbb{Z}^{n} \hookrightarrow$ $(M, \omega) \rightarrow B$ via

$$
\chi_{*}=\mathfrak{l}^{-T},
$$

where $-T$ denotes inverse transposed (cf. equation (44)). However, the linear holonomy alone does not determine the symplectic reference bundle up to fibrewise symplectomorphism, as illustrated by the next simple example.

Example 3.11 (Some symplectic reference bundles Luk08, Sep10a). Let $\mathbb{R} / \mathbb{Z}, \mathbb{R} / 2 \mathbb{Z}$ be the integral affine manifolds obtained by taking the quotient of $\mathbb{R}$ by the lattices $\mathbb{Z}$ and $2 \mathbb{Z}$, which are affinely isomorphic, but not integrally affinely isomorphic. Their linear holonomies are trivial, so that their symplectic reference Lagrangian bundles are principal $S^{1}$-bundles with a section, i.e. they are globally trivial. Thus their topological reference bundles are isomorphic. However, the total spaces of their symplectic reference bundles are symplectomorphic to

$$
\left(\mathbb{R}^{2} / \mathbb{Z}^{2}, \omega_{1}\right),\left(\mathbb{R}^{2} / 2 \mathbb{Z} \oplus \mathbb{Z}, \omega_{2}\right)
$$

respectively, where $\omega_{1}, \omega_{2}$ are symplectic forms which descend from the standard symplectic form $\Omega$ obtained by considering $\mathbb{R}^{2} \cong \mathrm{T}^{*} \mathbb{R}$ (cf. [uk08). These two symplectic manifolds cannot be symplectomorphic since the total spaces have different volumes. This example can been refined to work in the case when the total spaces have the same volume (cf. Sep10a).

The idea that lies at the heart of Example 3.11 is that the symplectic reference bundle $\mathbb{R}^{n} / \mathbb{Z}^{n} \hookrightarrow$ $\left(\mathrm{T}^{*} B / P_{(B, \mathcal{A})}, \omega_{0}\right) \rightarrow B$ associated to an integral affine manifold $(B, \mathcal{A})$ contains information about the integral affine structure $\mathcal{A}$. This idea is further explored in Section 5. 
3.2. Almost Lagrangian bundles. In light of Section 3.1 it is now possible to state main question that this paper tackles.

Question. Let $(B, \mathcal{A})$ be an $n$-dimensional integral affine manifold with linear holonomy $\mathfrak{l}$, and let $\mathbb{R}^{n} / \mathbb{Z}^{n} \hookrightarrow$ $M \rightarrow B$ be an affine $\mathbb{R}^{n} / \mathbb{Z}^{n}$-bundle classified by the homotopy class of a map

$$
\chi: B \rightarrow \operatorname{BAff}\left(\mathbb{R}^{n} / \mathbb{Z}^{n}\right),
$$

which satisfies the condition of equation (5). What is the obstruction to endowing $M$ with a symplectic form $\omega$ which makes the bundle Lagrangian?

Definition 3.12 (Almost Lagrangian bundles). For a fixed $n$-dimensional integral affine manifold $(B, \mathcal{A})$ with linear holonomy $\mathfrak{l}$, the affine $\mathbb{R}^{n} / \mathbb{Z}^{n}$-bundles over $B$ whose monodromy satisfies the condition of equation (5) are called almost Lagrangian.

Remark 3.13 (Classification of almost Lagrangian bundles). The isomorphism classes of almost Lagrangian bundles over an $n$-dimensional integral affine manifold $(B, \mathcal{A})$ with linear holonomy $\mathfrak{l}$ are in $1-1$ correspondence with elements of $\mathrm{H}^{2}\left(B ; \mathbb{Z}_{\mathfrak{l}-T}^{n}\right)$ (cf. Remark 3.2). The almost Lagrangian bundle corresponding to $0 \in \mathrm{H}^{2}\left(B ; \mathbb{Z}_{\mathfrak{l}^{-T}}^{n}\right)$ is necessarily Lagrangian and the symplectic form on its total space can always be chosen so as to admit a Lagrangian section (cf. Remark 3.4).

Remark 3.14 (Local trivialisations of almost Lagrangian bundles). Fix an almost Lagrangian bundle $\mathbb{R}^{n} / \mathbb{Z}^{n} \hookrightarrow M \rightarrow B$ over $(B, \mathcal{A})$ and denote by $P$ its period lattice bundle

$$
\mathrm{H}_{1}\left(\mathbb{R}^{n} / \mathbb{Z}^{n} ; \mathbb{Z}\right) \hookrightarrow P \rightarrow B,
$$

whose isomorphism class is determined by a homomorphism

$$
\pi_{1}(B) \rightarrow \operatorname{Aut}\left(\mathbb{Z}^{n}\right) \cong \mathrm{GL}(n ; \mathbb{Z}),
$$

which, up to a choice of basepoint, equals the inverse transposed $\mathfrak{l}^{-T}$ of the linear holonomy of $(B, \mathcal{A})$ by definition. If $P_{(B, \mathcal{A})} \rightarrow B$ denotes the period lattice bundle associated to $(B, \mathcal{A})$ (cf. Definition 3.8), then there is an isomorphism

$$
P \cong P_{(B, \mathcal{A})} .
$$

Let $\mathcal{U}=\left\{U_{\alpha}\right\}$ be a good (in the sense of Leray) open cover of $B$ by integral affine coordinate neighbourhoods. The restriction

$$
\mathbb{R}^{n} / \mathbb{Z}^{n} \hookrightarrow \pi^{-1}\left(U_{\alpha}\right) \rightarrow U_{\alpha}
$$

is an almost Lagrangian bundle. Since $U_{\alpha}$ is contractible, the above bundle is trivial and therefore there exists a section $s_{\alpha}: U_{\alpha} \rightarrow \pi^{-1}\left(U_{\alpha}\right)$. Fix such a section. Since $P_{\alpha}=\left.P\right|_{U_{\alpha}} \rightarrow U_{\alpha}$ is trivial, the above identification can be extended to a trivialisation

$$
\pi^{-1}\left(U_{\alpha}\right) \cong\left(P_{\alpha} \otimes_{\mathbb{Z}} \mathbb{R}\right) / P_{\alpha}
$$

using the section $s_{\alpha}$. The isomorphism of equation (6) extends to yield an isomorphism

$$
P \otimes_{\mathbb{Z}} \mathbb{R} \cong P_{(B, \mathcal{A})} \otimes_{\mathbb{Z}} \mathbb{R} \cong \mathrm{T}^{*} B,
$$

where the second isomorphism follows from the definition of $P_{(B, \mathcal{A})}$. In particular, the restriction of this isomorphism to $\pi^{-1}\left(U_{\alpha}\right)$ defines a trivialisation

$$
\Upsilon_{\alpha}: \pi^{-1}\left(U_{\alpha}\right) \rightarrow \mathrm{T}^{*} U_{\alpha} /\left.P_{(B, \mathcal{A})}\right|_{U_{\alpha}}
$$

which maps the section $s_{\alpha}$ to the zero section of $\mathrm{T}^{*} U_{\alpha} /\left.P_{(B, \mathcal{A})}\right|_{U_{\alpha}} \rightarrow U_{\alpha}$. Note that if $\omega_{0}$ denotes the symplectic form making the bundle

$$
\mathrm{T}^{*} B / P_{(B, \mathcal{A})} \rightarrow B
$$

into the symplectic reference bundle associated to $(B, \mathcal{A})$, and $\omega_{0, \alpha}$ is its restriction to $\mathrm{T}^{*} U_{\alpha} /\left.P_{(B, \mathcal{A})}\right|_{U_{\alpha}}$, then

$$
\Upsilon_{\alpha}^{*} \omega_{0, \alpha}
$$

makes the bundle of equation (77) Lagrangian. Furthermore, the section $s_{\alpha}$ is also Lagrangian. 
If $\left(\mathbf{a}_{\alpha}, \boldsymbol{\theta}_{\alpha}\right)$ denote action-angle coordinates on $\mathrm{T}^{*} U_{\alpha} /\left.P_{(B, \mathcal{A})}\right|_{U_{\alpha}}$, the transition functions

$$
\varphi_{\beta \alpha}=\Upsilon_{\beta} \circ \Upsilon_{\alpha}^{-1}: \mathrm{T}^{*}\left(U_{\alpha} \cap U_{\beta}\right) /\left.P_{(B, \mathcal{A})}\right|_{U_{\alpha} \cap U_{\beta}} \rightarrow \mathrm{T}^{*}\left(U_{\alpha} \cap U_{\beta}\right) /\left.P_{(B, \mathcal{A})}\right|_{U_{\alpha} \cap U_{\beta}}
$$

for the above choice of trivialisations take the familiar form (cf. equation (4))

$$
\varphi_{\beta \alpha}\left(\mathbf{a}_{\alpha}, \boldsymbol{\theta}_{\alpha}\right)=\left(A_{\beta \alpha} \mathbf{a}_{\alpha}+\mathbf{d}_{\beta \alpha}, A_{\beta \alpha}^{-T} \boldsymbol{\theta}_{\alpha}+\mathbf{g}_{\beta \alpha}\left(\mathbf{a}_{\alpha}\right)\right)
$$

where the first component comes from an affine change of coordinates on $U_{\alpha} \cap U_{\beta}$, and the linear part of the second component is determined by the transition functions for $P_{(B, \mathcal{A})} \rightarrow B$ by construction. Note that the maps $\varphi_{\beta \alpha}$ are not necessarily fibrewise symplectomorphisms as the locally defined forms $\Upsilon_{\alpha}^{*} \omega_{0, \alpha}$ do not necessarily patch together.

Remark 3.15 (Equivalent definition of almost Lagrangian bundles [DD87]). Fix an $n$-dimensional integral affine manifold $(B, \mathcal{A})$ with linear holonomy $\mathfrak{l}$, and let $P_{(B, \mathcal{A})} \subset \mathrm{T}^{*} B$ denote its associated period lattice bundle. Denote by $\mathcal{P}$ the sheaf of smooth sections of the projection $P_{(B, \mathcal{A})} \rightarrow B$. Note that $P_{(B, \mathcal{A})}$ is isomorphic to the period lattice bundle of any Lagrangian bundle over $B$ whose monodromy equals $\mathfrak{l}^{-T}$, as proved in Dui80. Thus there is an isomorphism of cohomology groups

$$
\mathrm{H}^{i}(B ; \mathcal{P}) \cong \mathrm{H}^{i}\left(B ; \mathbb{Z}_{\mathfrak{l}^{-T}}^{n}\right)
$$

for all $i$. The sheaf $\mathcal{P}$ fits into a short exact sequence (cf. Dui80])

$$
0 \rightarrow \mathcal{P} \rightarrow \mathcal{C}^{\infty}\left(\mathrm{T}^{*} B\right) \rightarrow \mathcal{C}^{\infty}\left(\mathrm{T}^{*} B / P_{(B, \mathcal{A})}\right) \rightarrow 0,
$$

where $\mathcal{C}^{\infty}\left(\mathrm{T}^{*} B\right)$ and $\mathcal{C}^{\infty}\left(\mathrm{T}^{*} B / P_{(B, \mathcal{A})}\right)$ are the sheaves of smooth sections of the cotangent bundle $\mathrm{T}^{*} B \rightarrow$ $B$ and of the topological reference bundle $\mathrm{T}^{*} B / P_{(B, \mathcal{A})} \rightarrow B$ respectively. The long exact sequence in cohomology induced by equation (9) collapses to isomorphisms

$$
\mathrm{H}^{i}\left(B ; \mathcal{C}^{\infty}\left(\mathrm{T}^{*} B / P_{(B, \mathcal{A})}\right)\right) \cong \mathrm{H}^{i+1}(B ; \mathcal{P})
$$

for $i \geq 1$, since $\mathcal{C}^{\infty}\left(\mathrm{T}^{*} B\right)$ is a fine sheaf. In particular,

$$
\mathrm{H}^{1}\left(B ; \mathcal{C}^{\infty}\left(\mathrm{T}^{*} B / P_{(B, \mathcal{A})}\right)\right) \cong \mathrm{H}^{2}(B ; \mathcal{P}) .
$$

Hence

$$
\mathrm{H}^{1}\left(B ; \mathcal{C}^{\infty}\left(\mathrm{T}^{*} B / P_{(B, \mathcal{A})}\right)\right) \cong \mathrm{H}^{2}\left(B ; \mathbb{Z}_{\mathfrak{l}^{-T}}^{n}\right) .
$$

The group $\mathrm{H}^{1}\left(B ; \mathcal{C}^{\infty}\left(\mathrm{T}^{*} B / P_{(B, \mathcal{A})}\right)\right)$ classifies the isomorphism classes of bundles over $B$ which are locally isomorphic to $\mathrm{T}^{*} B / P_{(B, \mathcal{A})} \rightarrow B$ and have structure sheaf $\mathcal{C}^{\infty}\left(\mathrm{T}^{*} B / P_{(B, \mathcal{A})}\right)$ (cf. [DD87, Gro58). In light of Remark 3.13 and equation (10), an almost Lagrangian bundle satisfies this condition and, conversely, any bundle over $B$ with the above properties is almost Lagrangian. This is the point of view taken in DD87, and, more generally, in other works in the literature, e.g. [Dui80, Luk08, Zun03.

Remark 3.16 (Relation to integrable systems [FS07]). Almost Lagrangian bundles are the correct geometric setting for studying the type of generalised Liouville integrability that Fassò and Sansonetto consider in [FS07. It can be shown that the total space of an almost Lagrangian bundle admits an appropriate nondegenerate 2 -form with respect to which the fibres are maximally isotropic submanifolds. This work will appear in SS11.

Not all almost Lagrangian bundles are Lagrangian. Fix an $n$-dimensional integral affine manifold $(B, \mathcal{A})$ with linear holonomy $\mathfrak{l}$, and let $P_{(B, \mathcal{A})} \subset \mathrm{T}^{*} B$ be the period lattice bundle associated to $(B, \mathcal{A})$ (cf. Definition 3.8). The sheaf of smooth sections $\mathcal{P}$ of $P_{(B, \mathcal{A})} \rightarrow B$ fits in a short exact sequence

$$
0 \rightarrow \mathcal{P} \rightarrow \mathcal{Z}\left(\mathrm{T}^{*} B\right) \rightarrow \mathcal{Z}\left(\mathrm{T}^{*} B / P_{(B, \mathcal{A})}\right) \rightarrow 0
$$

where $\mathcal{Z}\left(\mathrm{T}^{*} B\right)$ and $\mathcal{Z}\left(\mathrm{T}^{*} B / P_{(B, \mathcal{A})}\right)$ are the sheaves of closed sections of $\mathrm{T}^{*} B \rightarrow B$ and $\mathrm{T}^{*} B / P_{(B, \mathcal{A})} \rightarrow B$ respectively. The induced long exact sequence in cohomology groups yields a homomorphism

$$
\mathcal{D}_{(B, \mathcal{A})}: \mathrm{H}^{2}(B ; \mathcal{P}) \rightarrow \mathrm{H}^{2}\left(B ; \mathcal{Z}\left(\mathrm{T}^{*} B\right)\right)
$$

where the subscript denotes the dependence upon the integral affine structure of the manifold $B$. This homomorphism depends on $\mathcal{A}$ as the latter determines $P_{(B, \mathcal{A})}$ as a Lagrangian submanifold of $\left(\mathrm{T}^{*} B, \Omega\right)$ (cf. Definition 3.8). By the Poincaré Lemma (cf. Wei71]),

$$
\mathrm{H}^{2}\left(B ; \mathcal{Z}\left(\mathrm{T}^{*} B\right)\right) \cong \mathrm{H}^{3}(B ; \mathbb{R}),
$$


where $\mathrm{H}^{3}(B ; \mathbb{R})$ denotes cohomology with real coefficients, and the isomorphism between this cohomology theory and the cohomology with coefficients in the constant sheaf $\mathcal{R}$ over $B$ with $\mathbb{R}$ coefficients is used tacitly. Hence there is a homomorphism

$$
\mathcal{D}_{(B, \mathcal{A})}: \mathrm{H}^{2}(B ; \mathcal{P}) \rightarrow \mathrm{H}^{3}(B ; \mathbb{R}) .
$$

Theorem 3.3 (Dazord and Delzant [DD87]). Let $\mathbb{R}^{n} / \mathbb{Z}^{n} \hookrightarrow M \rightarrow B$ be an almost Lagrangian bundle over $(B, \mathcal{A})$ with linear holonomy $\mathfrak{l}$, and let

$$
c \in \mathrm{H}^{2}\left(B ; \mathbb{Z}_{\mathfrak{l}^{-T}}^{n}\right) \cong \mathrm{H}^{2}(B ; \mathcal{P})
$$

be its Chern class. Then $\mathbb{R}^{n} / \mathbb{Z}^{n} \hookrightarrow M \rightarrow B$ is Lagrangian if and only if

$$
\mathcal{D}_{(B, \mathcal{A})} c=0 .
$$

The rest of this paper is devoted to proving that the homomorphism $\mathcal{D}_{(B, \mathcal{A})}$ is related to a differential on the $\mathrm{E}_{2}$-page of the Leray-Serre spectral sequence of the topological universal Lagrangian bundles constructed in Section 2, This observation allows to give a different proof of Theorem 3.3, which highlights the importance of integral affine geometry.

\section{A Spectral Sequence}

4.1. A preparatory lemma. In this section, the universal Lagrangian bundle is identified as the equivariant equivalent of the universal bundle for principal $\mathbb{R}^{n} / \mathbb{Z}^{n}$-bundles. Free monodromy of an affine $\mathbb{R}^{n} / \mathbb{Z}^{n}$-bundle is the obstruction for it to be a principal $\mathbb{R}^{n} / \mathbb{Z}^{n}$-bundle. Therefore, if $\tau: \mathrm{B} \mathbb{R}^{n} / \mathbb{Z}^{n} \rightarrow \mathrm{BAff}\left(\mathbb{R}^{n} / \mathbb{Z}^{n}\right)$ denotes the bundle induced by the inclusion $\tau: \mathbb{R}^{n} / \mathbb{Z}^{n} \hookrightarrow \mathrm{Aff}_{\mathbb{Z}}\left(\mathbb{R}^{n}\right)$, then the following lemma holds.

Lemma 4.1. The bundle

$$
\mathbb{R}^{n} / \mathbb{Z}^{n} \hookrightarrow \tau^{*} \operatorname{BGL}(n ; \mathbb{Z}) \rightarrow \mathrm{BR}^{n} / \mathbb{Z}^{n}
$$

obtained by pulling back the universal Lagrangian bundle along the universal covering map $\tau: \mathrm{BR}^{n} / \mathbb{Z}^{n} \rightarrow$ $\operatorname{BAff}\left(\mathbb{R}^{n} / \mathbb{Z}^{n}\right)$ is a universal bundle for principal $\mathbb{R}^{n} / \mathbb{Z}^{n}$-bundles.

Remark 4.1. By functoriality of the Chern class, the obstruction to the existence of a section of the bundle of equation (11) is given by

$$
\tau^{*} c_{U} \in \mathrm{H}^{2}\left(\mathrm{~B} \mathbb{R}^{n} / \mathbb{Z}^{n} ; \mathbb{Z}_{(\mathrm{ido} \tau)_{*}}^{n}\right)
$$

where $c_{U}$ denotes the universal Chern class. Note that $\pi_{1}\left(\mathrm{BR}^{n} / \mathbb{Z}^{n}\right)$ is trivial, so that the above coefficient system is just the constant $\mathbb{Z}^{n}$-system of coefficients on $\mathrm{B} \mathbb{R}^{n} / \mathbb{Z}^{n}$. Therefore

$$
\tau^{*} c_{U}=c_{\mathbb{R}^{n} / \mathbb{Z}^{n}}
$$

where $c_{\mathbb{R}^{n}} / \mathbb{Z}^{n}$ is the obstruction to the existence of a section for the bundle

$$
\mathbb{R}^{n} / \mathbb{Z}^{n} \hookrightarrow \mathrm{E} \mathbb{R}^{n} / \mathbb{Z}^{n} \rightarrow \mathrm{B} \mathbb{R}^{n} / \mathbb{Z}^{n}
$$

4.2. The spectral sequence of a universal Lagrangian bundle. In this section the methods of [CV66] are adapted to prove that some differential on the $\mathrm{E}_{2}$-page of the Leray-Serre spectral sequence of the universal Lagrangian bundle is given (up to some isomorphisms) by taking cup products with the universal Chern class

$$
c_{U} \in \mathrm{H}^{2}\left(\operatorname{BAff}\left(\mathbb{R}^{n} / \mathbb{Z}^{n}\right) ; \mathbb{Z}_{\mathrm{id}_{*}}^{n}\right) .
$$

This result is crucial to study the problem of constructing Lagrangian bundles over a fixed integral affine manifold.

Given a spectral sequence, the main idea in [CV66] is to use auxiliary spectral sequences to reduce the problem of determining differentials on the $\mathrm{E}_{2}$-page of the original spectral sequence to a simpler one. While CV66 deals with the cohomology of group extensions and, in particular, with abelian extensions, the case of the universal Lagrangian bundle and, more generally, of affine $\mathbb{R}^{n} / \mathbb{Z}^{n}$-bundles can be thought of as a natural generalisation. This is because the interesting part of the long exact sequence in homotopy for an affine $\mathbb{R}^{n} / \mathbb{Z}^{n}$-bundle $\mathbb{R}^{n} / \mathbb{Z}^{n} \hookrightarrow M \rightarrow B$ is

$$
0 \rightarrow \pi_{2} M \rightarrow \pi_{2} B \rightarrow \pi_{1} \mathbb{R}^{n} / \mathbb{Z}^{n} \rightarrow \pi_{1} M \rightarrow \pi_{1} B \rightarrow 1 .
$$


Auxiliary spectral sequences. The $\mathrm{E}_{2}$-page of the Leray-Serre spectral sequence for the universal Lagrangian bundle

$$
\mathbb{R}^{n} / \mathbb{Z}^{n} \hookrightarrow \operatorname{BAff}\left(\mathbb{R}^{n} / \mathbb{Z}^{n}\right) \rightarrow \operatorname{BGL}(n ; \mathbb{Z})
$$

with $\mathbb{Z}$ coefficients is denoted by

$$
\mathrm{E}_{2}^{p, q} \cong \mathrm{H}^{p}\left(\mathrm{BAff}\left(\mathbb{R}^{n} / \mathbb{Z}^{n}\right) ; \mathrm{H}^{q}\left(\mathbb{R}^{n} / \mathbb{Z}^{n} ; \mathbb{Z}\right)_{\rho_{q}}\right) .
$$

The homomorphism

$$
\rho_{q}: \pi_{1}\left(\operatorname{BAff}\left(\mathbb{R}^{n} / \mathbb{Z}^{n}\right)\right) \rightarrow \operatorname{Aut}\left(\mathrm{H}^{q}\left(\mathbb{R}^{n} / \mathbb{Z}^{n} ; \mathbb{Z}\right)\right)
$$

classifies the local coefficient system defined by replacing each fibre $\mathbb{R}^{n} / \mathbb{Z}^{n}$ of the universal Lagrangian bundle with its $q$-th cohomology group with integer coefficients $\mathrm{H}^{q}\left(\mathbb{R}^{n} / \mathbb{Z}^{n} ; \mathbb{Z}\right)$. Henceforth, fix a basepoint in BAff $\left(\mathbb{R}^{n} / \mathbb{Z}^{n}\right)$, so that the above homomorphisms are also fixed. Denote by

$$
\breve{\rho}_{1}: \pi_{1}\left(\operatorname{BAff}\left(\mathbb{R}^{n} / \mathbb{Z}^{n}\right)\right) \rightarrow \operatorname{Aut}\left(\mathrm{H}_{1}\left(\mathbb{R}^{n} / \mathbb{Z}^{n} ; \mathbb{Z}\right)\right)
$$

the homomorphism classifying the local coefficient system with fibre $\mathrm{H}_{1}\left(\mathbb{R}^{n} / \mathbb{Z}^{n} ; \mathbb{Z}\right)$ over BAff $\left(\mathbb{R}^{n} / \mathbb{Z}^{n}\right)$. Following [CV66], introduce auxiliary spectral sequences, whose $\mathrm{E}_{2}$-pages are given by

$$
\begin{aligned}
& \overline{\mathrm{E}}_{2}^{p, q} \cong \mathrm{H}^{p}\left(\mathrm{BAff}\left(\mathbb{R}^{n} / \mathbb{Z}^{n}\right) ; \mathrm{H}^{q}\left(\mathbb{R}^{n} / \mathbb{Z}^{n} ; \mathrm{H}^{1}\left(\mathbb{R}^{n} / \mathbb{Z}^{n} ; \mathbb{Z}\right)\right)_{\bar{\rho}_{q}}\right), \\
& \hat{\mathrm{E}}_{2}^{p, q} \cong \mathrm{H}^{p}\left(\operatorname{BAff}\left(\mathbb{R}^{n} / \mathbb{Z}^{n}\right) ; \mathrm{H}^{q}\left(\mathbb{R}^{n} / \mathbb{Z}^{n} ; \mathrm{H}_{1}\left(\mathbb{R}^{n} / \mathbb{Z}^{n} ; \mathbb{Z}\right)\right)_{\hat{\rho}_{q}}\right),
\end{aligned}
$$

where the above local coefficient systems are given by

$$
\begin{aligned}
& \bar{\rho}_{q}=\rho_{q} \otimes \rho_{1}: \pi_{1}\left(\operatorname{BAff}\left(\mathbb{R}^{n} / \mathbb{Z}^{n}\right)\right) \rightarrow \operatorname{Aut}\left(\mathrm{H}^{q}\left(\mathbb{R}^{n} / \mathbb{Z}^{n} ; \mathbb{Z}\right) \otimes \mathrm{H}^{1}\left(\mathbb{R}^{n} / \mathbb{Z}^{n} ; \mathbb{Z}\right)\right), \\
& \hat{\rho}_{q}=\rho_{q} \otimes \breve{\rho}_{1}: \pi_{1}\left(\operatorname{BAff}\left(\mathbb{R}^{n} / \mathbb{Z}^{n}\right)\right) \rightarrow \operatorname{Aut}\left(\mathrm{H}^{q}\left(\mathbb{R}^{n} / \mathbb{Z}^{n} ; \mathbb{Z}\right) \otimes \mathrm{H}_{1}\left(\mathbb{R}^{n} / \mathbb{Z}^{n} ; \mathbb{Z}\right)\right),
\end{aligned}
$$

via the isomorphisms

$$
\begin{aligned}
& \mathrm{H}^{q}\left(\mathbb{R}^{n} / \mathbb{Z}^{n} ; \mathrm{H}^{1}\left(\mathbb{R}^{n} / \mathbb{Z}^{n} ; \mathbb{Z}\right)\right) \cong \mathrm{H}^{q}\left(\mathbb{R}^{n} / \mathbb{Z}^{n} ; \mathbb{Z}\right) \otimes \mathrm{H}^{1}\left(\mathbb{R}^{n} / \mathbb{Z}^{n} ; \mathbb{Z}\right), \\
& \mathrm{H}^{q}\left(\mathbb{R}^{n} / \mathbb{Z}^{n} ; \mathrm{H}_{1}\left(\mathbb{R}^{n} / \mathbb{Z}^{n} ; \mathbb{Z}\right)\right) \cong \mathrm{H}^{q}\left(\mathbb{R}^{n} / \mathbb{Z}^{n} ; \mathbb{Z}\right) \otimes \mathrm{H}_{1}\left(\mathbb{R}^{n} / \mathbb{Z}^{n} ; \mathbb{Z}\right),
\end{aligned}
$$

induced by the universal coefficient theorem. These spectral sequences are henceforth referred to as the Leray-Serre spectral sequences with $\mathrm{H}^{1}\left(\mathbb{R}^{n} / \mathbb{Z}^{n} ; \mathbb{Z}\right)$ and $\mathrm{H}_{1}\left(\mathbb{R}^{n} / \mathbb{Z}^{n} ; \mathbb{Z}\right)$ coefficients respectively.

There is a pairing

$$
\overline{\mathrm{E}}_{2}^{p, q} \otimes_{\mathbb{Z}} \hat{\mathrm{E}}_{2}^{p^{\prime}, q^{\prime}} \rightarrow \mathrm{E}_{2}^{p+p^{\prime}, q+q^{\prime}}
$$

induced by taking cup products and by the standard duality

$$
\mathrm{H}^{1}\left(\mathbb{R}^{n} / \mathbb{Z}^{n} ; \mathbb{Z}\right) \otimes_{\mathbb{Z}} \mathrm{H}_{1}\left(\mathbb{R}^{n} / \mathbb{Z}^{n} ; \mathbb{Z}\right) \rightarrow \mathbb{Z},
$$

called the auxiliary pairing associated to the universal Lagrangian bundle.

If $\mathrm{d}^{(2)}, \overline{\mathrm{d}}^{(2)}, \hat{\mathrm{d}}^{(2)}$ denote the differentials on the $\mathrm{E}_{2}$-page of the spectral sequences $\mathrm{E}_{2}^{*, *}, \overline{\mathrm{E}}_{2}^{*, *}, \hat{\mathrm{E}}_{2}^{*, *}$ respectively, there is the multiplicative formula

$$
\mathrm{d}^{(2)}(x \cdot y)=\overline{\mathrm{d}}^{(2)}(x) \cdot y+(-1)^{p+q} x \cdot \hat{\mathrm{d}}^{(2)}(y),
$$

where $x \in \hat{\mathrm{E}}_{2}^{p, q}, y \in \overline{\mathrm{E}}_{2}^{p^{\prime}, q^{\prime}}$ and $x \cdot y$ denotes the auxiliary pairing between $x$ and $y$.

As shown in [CV66], there is an isomorphism

$$
\theta: \mathrm{E}_{2}^{p, 1} \rightarrow \overline{\mathrm{E}}_{2}^{p, 0}
$$

induced by

$$
\mathrm{H}^{1}\left(\mathbb{R}^{n} / \mathbb{Z}^{n} ; \mathbb{Z}\right) \rightarrow \mathrm{H}^{0}\left(\mathbb{R}^{n} / \mathbb{Z}^{n} ; \mathrm{H}^{1}\left(\mathbb{R}^{n} / \mathbb{Z}^{n} ; \mathbb{Z}\right)\right)
$$

The identity map

$$
\text { id }: \mathrm{H}_{1}\left(\mathbb{R}^{n} / \mathbb{Z}^{n} ; \mathbb{Z}\right) \rightarrow \mathrm{H}_{1}\left(\mathbb{R}^{n} / \mathbb{Z}^{n} ; \mathbb{Z}\right)
$$

defines an element $g^{1} \in \mathrm{H}^{1}\left(\mathbb{R}^{n} / \mathbb{Z}^{n} ; \mathrm{H}_{1}\left(\mathbb{R}^{n} / \mathbb{Z}^{n} ; \mathbb{Z}\right)\right)$, since

$$
\mathrm{H}^{1}\left(\mathbb{R}^{n} / \mathbb{Z}^{n} ; \mathrm{H}_{1}\left(\mathbb{R}^{n} / \mathbb{Z}^{n} ; \mathbb{Z}\right)\right) \cong \operatorname{Hom}\left(\mathrm{H}_{1}\left(\mathbb{R}^{n} / \mathbb{Z}^{n} ; \mathbb{Z}\right) ; \mathrm{H}_{1}\left(\mathbb{R}^{n} / \mathbb{Z}^{n} ; \mathbb{Z}\right)\right) .
$$


The element $g^{1}$, in turn, defines an element

$$
f^{1} \in \hat{\mathrm{E}}_{2}^{0,1} \cong \mathrm{H}^{0}\left(\mathrm{BAff}\left(\mathbb{R}^{n} / \mathbb{Z}^{n}\right) ; \mathrm{H}^{1}\left(\mathbb{R}^{n} / \mathbb{Z}^{n} ; \mathrm{H}_{1}\left(\mathbb{R}^{n} / \mathbb{Z}^{n} ; \mathbb{Z}\right)\right)_{\hat{\rho}_{1}}\right),
$$

since $g^{1}$ is fixed by the action of $\pi_{1} \operatorname{BAff}\left(\mathbb{R}^{n} / \mathbb{Z}^{n}\right)$ defined by $\hat{\rho}_{1}$. The following two propositions are stated below without proof.

Proposition 4.2 (Proposition 2.1 CV66]). Let $x \in \mathrm{E}_{2}^{p, 1}$. Then

$$
x=\theta(x) \cdot f^{1},
$$

where $\cdot$ denotes the auxiliary pairing defined above.

Proposition 4.3 (Proposition 2.2 CV66]). Let $x \in \mathrm{E}_{2}^{p, 1}$. Then

$$
\mathrm{d}^{(2)}(x)=(-1)^{p+1} \theta(x) \cdot \hat{\mathrm{d}}^{(2)}\left(f^{1}\right) .
$$

Proposition 4.3 reduces the problem of determining $\mathrm{d}^{(2)}$ to that of determining $\hat{\mathrm{d}}^{(2)}\left(f^{1}\right)$, which depends on the universal Chern class $c_{U}$ : this is the content of Theorem 4.6.

Before computing $\hat{\mathrm{d}}^{(2)}\left(f^{1}\right)$, it is useful to compute the corresponding differential for the bundle

$$
\mathbb{R}^{n} / \mathbb{Z}^{n} \hookrightarrow \mathrm{ER}^{n} / \mathbb{Z}^{n} \rightarrow \mathrm{B} \mathbb{R}^{n} / \mathbb{Z}^{n},
$$

which, in light of Lemma 4.1 is isomorphic to the pull-back of the universal Lagrangian bundle along the universal covering map $\tau: \mathrm{BR}^{n} / \mathbb{Z}^{n} \rightarrow \mathrm{BAff}\left(\mathbb{R}^{n} / \mathbb{Z}^{n}\right)$. Let

$$
\hat{\mathrm{E}}_{2, \mathbb{R}^{n} / \mathbb{Z}^{n}}^{p, q} \mathrm{H}^{p}\left(\mathrm{~B} \mathbb{R}^{n} / \mathbb{Z}^{n} ; \mathrm{H}^{q}\left(\mathbb{R}^{n} / \mathbb{Z}^{n} ; \mathrm{H}_{1}\left(\mathbb{R}^{n} / \mathbb{Z}^{n} ; \mathbb{Z}\right)\right)\right)
$$

denote the $\mathrm{E}_{2}$-page of the Leray-Serre spectral sequence for the bundle (17) with $\mathrm{H}_{1}\left(\mathbb{R}^{n} / \mathbb{Z}^{n} ; \mathbb{Z}\right)$ coefficients, and let $\hat{\mathrm{d}}_{\mathbb{R}^{n} / \mathbb{Z}^{n}}^{(2)}$ denote the corresponding differential. Let $f_{\mathbb{R}^{n} / \mathbb{Z}^{n}}^{1} \in \hat{\mathrm{E}}_{2, \mathbb{R}^{n} / \mathbb{Z}^{n}}^{0,}$ be the element arising from the identity map

$$
\text { id : } \mathrm{H}_{1}\left(\mathbb{R}^{n} / \mathbb{Z}^{n} ; \mathbb{Z}\right) \rightarrow \mathrm{H}_{1}\left(\mathbb{R}^{n} / \mathbb{Z}^{n} ; \mathbb{Z}\right)
$$

as above. In fact, in light of Lemma 4.1, the relation between $f^{1}$ and $f_{\mathbb{R}^{n} / \mathbb{Z}^{n}}^{1}$ is given by

$$
f_{\mathbb{R}^{n} / \mathbb{Z}^{n}}^{1}=\tau^{*} f^{1}
$$

Let

$$
\psi_{\mathbb{R}^{n} / \mathbb{Z}^{n}}: \mathrm{H}^{2}\left(\mathrm{BR}^{n} / \mathbb{Z}^{n} ; \mathrm{H}_{1}\left(\mathbb{R}^{n} / \mathbb{Z}^{n} ; \mathbb{Z}\right)\right) \rightarrow \hat{\mathrm{E}}_{2, \mathbb{R}^{n} / \mathbb{Z}^{n}}^{2,0}
$$

be the isomorphism arising via an identification

$$
\mathrm{H}^{0}\left(\mathbb{R}^{n} / \mathbb{Z}^{n} ; \mathrm{H}_{1}\left(\mathbb{R}^{n} / \mathbb{Z}^{n} ; \mathbb{Z}\right)\right) \cong \mathrm{H}_{1}\left(\mathbb{R}^{n} / \mathbb{Z}^{n} ; \mathbb{Z}\right)
$$

The following lemma computes the image of $f_{\mathbb{R}^{n} / \mathbb{Z}^{n}}^{1}$ under the differential $\hat{\mathrm{d}}_{\mathbb{R}^{n} / \mathbb{Z}^{n}}^{(2)}$. This is a well-known result (e.g. Gei92]), but a proof is included for completeness.

\section{Lemma 4.4.}

$$
\hat{\mathrm{d}}_{\mathbb{R}^{n} / \mathbb{Z}^{n}}^{(2)}\left(f_{\mathbb{R}^{n} / \mathbb{Z}^{n}}^{1}\right)=\psi\left(c_{\mathbb{R}^{n} / \mathbb{Z}^{n}}\right),
$$

where $c_{\mathbb{R}^{n} / \mathbb{Z}^{n}} \in \mathrm{H}^{2}\left(\mathrm{BR}^{n} / \mathbb{Z}^{n} ; \mathrm{H}_{1}\left(\mathbb{R}^{n} / \mathbb{Z}^{n} ; \mathbb{Z}\right)\right)$ is the Chern class of the universal bundle $\mathbb{R}^{n} / \mathbb{Z}^{n} \hookrightarrow \mathrm{ER}^{n} / \mathbb{Z}^{n} \rightarrow$ $\mathrm{BR}^{n} / \mathbb{Z}^{n}$.

Proof. By functoriality of the Chern class, it suffices to prove the result when $n=1$. In this case, the universal bundle is isomorphic (up to homotopy) to

$$
S^{1} \hookrightarrow S^{\infty} \rightarrow \mathbb{C P}^{\infty}
$$

Since $S^{\infty}$ is contractible, the differential

$$
\hat{\mathrm{d}}_{S^{1}}^{(2)}: \hat{\mathrm{E}}_{2, S^{1}}^{0,1} \rightarrow \hat{\mathrm{E}}_{2, S^{1}}^{2,0}
$$

is an isomorphism. In particular, since $f_{S^{1}}^{1} \in \hat{\mathrm{E}}_{2, S^{1}}^{0,1}$ is a generator, $\hat{\mathrm{d}}_{S^{1}}^{(2)}\left(f_{S^{1}}^{1}\right)$ is a generator of $\hat{\mathrm{E}}_{2, S^{1}}^{2,0}$, and thus equal to $\pm \psi_{S^{1}}\left(c_{S^{1}}\right)$. In fact, the normalisation axiom for the Chern classes implies that

$$
\hat{\mathrm{d}}_{S^{1}}^{(2)}\left(f_{S^{1}}^{1}\right)=\psi_{S^{1}}\left(c_{S^{1}}\right) .
$$


Let $\mathrm{E}_{2, \mathbb{R}^{n} / \mathbb{Z}^{n}}^{*, *}, \overline{\mathrm{E}}_{2, \mathbb{R}^{n} / \mathbb{Z}^{n}}^{*, *}$ denote the $\mathrm{E}_{2}$-pages of the Leray-Serre spectral sequences of

$$
\mathbb{R}^{n} / \mathbb{Z}^{n} \hookrightarrow \mathrm{ER}^{n} / \mathbb{Z}^{n} \rightarrow \mathrm{B} \mathbb{R}^{n} / \mathbb{Z}^{n}
$$

with $\mathbb{Z}$ and $\mathrm{H}^{1}\left(\mathbb{R}^{n} / \mathbb{Z}^{n} ; \mathbb{Z}\right)$ coefficients respectively, and denote by $\mathrm{d}_{\mathbb{R}^{n} / \mathbb{Z}^{n}}^{(2)}, \hat{\mathrm{d}}_{\mathbb{R}^{n} / \mathbb{Z}^{n}}^{(2)}$ their respective differentials. Lemma 4.4 and Proposition 4.3 can be combined to prove the following corollary, which is a version of Theorem 4.6 for principal $\mathbb{R}^{n} / \mathbb{Z}^{n}$-bundles.

Corollary 4.5. Let $x \in \mathrm{E}_{2, \mathbb{R}^{n} / \mathbb{Z}^{n}}^{p, 1}$. Its image $\mathrm{d}_{\mathbb{R}^{n} / \mathbb{Z}^{n}}^{(2)}(x) \in \mathrm{E}_{2, \mathbb{R}^{n} / \mathbb{Z}^{n}}^{p+2,0}$ is given by

$$
\mathrm{d}_{\mathbb{R}^{n} / \mathbb{Z}^{n}}^{(2)}(x)=(-1)^{p+1} \theta_{\mathbb{R}^{n} / \mathbb{Z}^{n}}(x) \cdot \mathbb{R}^{n} / \mathbb{Z}^{n} \psi_{\mathbb{R}^{n} / \mathbb{Z}^{n}}\left(c_{\mathbb{R}^{n} / \mathbb{Z}^{n}}\right),
$$

where $\theta_{\mathbb{R}^{n}} / \mathbb{Z}^{n}, \cdot \mathbb{R}^{n} / \mathbb{Z}^{n}$ are the analogues of the isomorphism of equation (16) and the auxiliary pairing of equation (14) for $\mathbb{R}^{n} / \mathbb{Z}^{n} \hookrightarrow \mathrm{ER}^{n} / \mathbb{Z}^{n} \rightarrow \mathrm{B} \mathbb{R}^{n} / \mathbb{Z}^{n}$.

The differential $\mathrm{d}^{(2)}$. The following theorem is one of the main results of this paper; it states that, up to isomorphisms, the differential

$$
\mathrm{d}^{(2)}: \mathrm{E}_{2}^{p, 1} \rightarrow \mathrm{E}_{2}^{p+2,0}
$$

is given by taking the cup product with the universal Chern class $c_{U}$.

Theorem 4.6. Let $x \in \mathrm{E}_{2}^{p, 1}$. Its image $\mathrm{d}^{(2)}(x) \in \mathrm{E}_{2}^{p+2,0} \cong \mathrm{H}^{p+2}\left(\operatorname{BAff}\left(\mathbb{R}^{n} / \mathbb{Z}^{n}\right) ; \mathbb{Z}\right)$ is given by

$$
\mathrm{d}^{(2)}(x)=(-1)^{p+1} \theta(x) \cdot \psi\left(c_{U}\right),
$$

where $\psi: \mathrm{H}^{2}\left(\mathrm{BAff}\left(\mathbb{R}^{n} / \mathbb{Z}^{n}\right) ; \mathbb{Z}_{\mathrm{id}_{*}}^{n}\right) \rightarrow \hat{\mathrm{E}}_{2}^{2,0}$ is the isomorphism induced by the identification

$$
\mathrm{H}^{0}\left(\mathbb{R}^{n} / \mathbb{Z}^{n} ; \mathrm{H}_{1}\left(\mathbb{R}^{n} / \mathbb{Z}^{n} ; \mathbb{Z}\right)\right) \cong \mathbb{Z}^{n}
$$

Proof. In light of Proposition 4.3, it suffices to show that

$$
\hat{\mathrm{d}}^{(2)}\left(f^{1}\right)=\psi\left(c_{U}\right),
$$

which is just the equivariant version of the result of Lemma 4.4. The idea of the proof is to use Lemma 4.4 and functoriality of the Leray-Serre spectral sequence to deduce the result.

By Lemma 4.1 the pull-back bundle

$$
\mathbb{R}^{n} / \mathbb{Z}^{n} \hookrightarrow \tau^{*} \operatorname{BGL}(n ; \mathbb{Z}) \rightarrow \mathrm{BR}^{n} / \mathbb{Z}^{n}
$$

is a universal bundle $\mathbb{R}^{n} / \mathbb{Z}^{n} \hookrightarrow \mathrm{ER}^{n} / \mathbb{Z}^{n} \rightarrow \mathrm{B} \mathbb{R}^{n} / \mathbb{Z}^{n}$. In particular, there is a commutative diagram

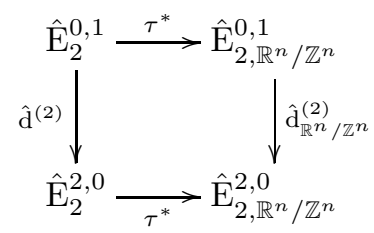

arising from functoriality of the Leray-Serre spectral sequence (cf. Section 6 in [McC85]). By Lemma 4.4,

$$
\hat{\mathrm{d}}_{\mathbb{R}^{n} / \mathbb{Z}^{n}}^{(2)} \circ \tau^{*}\left(f^{1}\right)=\psi_{\mathbb{R}^{n} / \mathbb{Z}^{n}}\left(c_{\mathbb{R}^{n} / \mathbb{Z}^{n}}\right),
$$

since $f_{\mathbb{R}^{n} / \mathbb{Z}^{n}}^{1}=\tau^{*} f^{1}$. Equation (23) and the commutativity of the diagram in equation (22) imply that

$$
\tau^{*} \circ \hat{\mathrm{d}}^{(2)}\left(f^{1}\right)=\psi_{\mathbb{R}^{n} / \mathbb{Z}^{n}}\left(c_{\mathbb{R}^{n}} / \mathbb{Z}^{n}\right) .
$$


Note that, by definition, the isomorphism $\psi$ is the equivariant version of $\psi_{\mathbb{R}^{n} / \mathbb{Z}^{n}}$, i.e. there is a commutative diagram

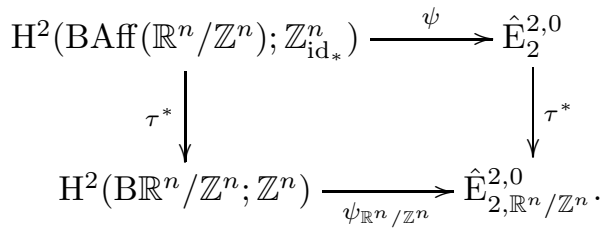

By equation (12), the commutative diagram (25) implies that

$$
\tau^{*} \circ \psi\left(c_{U}\right)=\psi_{\mathbb{R}^{n} / \mathbb{Z}^{n}}\left(c_{\mathbb{R}^{n} / \mathbb{Z}^{n}}\right) .
$$

In particular, combining equations (24) and (26), obtain that

$$
\hat{\mathrm{d}}^{(2)}\left(f^{1}\right)-\psi\left(c_{U}\right)=\mu \in \operatorname{ker} \tau^{*} .
$$

It therefore remains to show that $\mu=0$.

This is achieved in the following two steps.

Step 1 Prove that $\psi^{-1}(\mu)$ lies in the image of the homomorphism

$$
\operatorname{Lin}^{*}: \mathrm{H}^{2}\left(\operatorname{BGL}(n ; \mathbb{Z}) ; \mathbb{Z}_{\mathrm{id}_{*}^{G}}^{n}\right) \rightarrow \mathrm{H}^{2}\left(\operatorname{BAff}\left(\mathbb{R}^{n} / \mathbb{Z}^{n}\right) ; \mathbb{Z}_{\mathrm{id}_{*}}^{n}\right)
$$

induced by the fibration

$$
\text { Lin : } \operatorname{BAff}\left(\mathbb{R}^{n} / \mathbb{Z}^{n}\right) \rightarrow \operatorname{BGL}(n ; \mathbb{Z}) ;
$$

Step 2 Prove that $\psi^{-1}(\mu)$ lies in the kernel of the homomorphism

$$
\sigma^{*}: \mathrm{H}^{2}\left(\operatorname{BAff}\left(\mathbb{R}^{n} / \mathbb{Z}^{n}\right) ; \mathbb{Z}_{\mathrm{id}_{*}}^{n}\right) \rightarrow \mathrm{H}^{2}\left(\operatorname{BGL}(n ; \mathbb{Z}) ; \mathbb{Z}_{\mathrm{id}_{*}^{G}}^{n}\right)
$$

induced by the universal Lagrangian bundle

$$
\mathbb{R}^{n} / \mathbb{Z}^{n \complement} \mathrm{BGL}(n ; \mathbb{Z}) \stackrel{\sigma}{\longrightarrow} \mathrm{BAff}_{\mathbb{Z}}\left(\mathbb{R}^{n} / \mathbb{Z}^{n}\right) .
$$

Step 1. Recall that there is a fibration

$$
\mathrm{B} \mathbb{R}^{n} / \mathbb{Z}^{n \subseteq} \stackrel{\tau}{\longrightarrow} \mathrm{BAff}\left(\mathbb{R}^{n} / \mathbb{Z}^{n}\right) \stackrel{\text { Lin }}{\longrightarrow} \mathrm{BGL}(n ; \mathbb{Z})
$$

arising from the group $\mathrm{Aff}_{\mathbb{Z}}\left(\mathbb{R}^{n} / \mathbb{Z}^{n}\right)=\mathrm{GL}(n ; \mathbb{Z}) \ltimes \mathbb{R}^{n} / \mathbb{Z}^{n}$ (cf. Section 5 in Hus93]), and consider the bundle

$$
\mathbb{R}^{n} / \mathbb{Z}^{n} \hookrightarrow \sigma^{*} \operatorname{BGL}(n ; \mathbb{Z}) \rightarrow \operatorname{BGL}(n ; \mathbb{Z})
$$

obtained by pulling back the universal Lagrangian bundle along the map $\sigma: \operatorname{BGL}(n ; \mathbb{Z}) \rightarrow \operatorname{BAff}\left(\mathbb{R}^{n} / \mathbb{Z}^{n}\right)$ induced by the splitting $\sigma: \operatorname{GL}(n ; \mathbb{Z}) \rightarrow \operatorname{Aff}_{\mathbb{Z}}\left(\mathbb{R}^{n} / \mathbb{Z}^{n}\right)$. There is an associated system of local coefficients

$$
\mathrm{H}^{1}\left(\mathbb{R}^{n} / \mathbb{Z}^{n} ; \mathbb{Z}\right) \hookrightarrow \sigma^{*} P_{n} \rightarrow \operatorname{BGL}(n ; \mathbb{Z}),
$$

which is just the pull-back of the universal period lattice bundle $P_{n} \rightarrow \operatorname{BAff}\left(\mathbb{R}^{n} / \mathbb{Z}^{n}\right)$ along $\sigma$. The pull-back

$$
\mathrm{H}^{1}\left(\mathbb{R}^{n} / \mathbb{Z}^{n} ; \mathbb{Z}\right) \hookrightarrow \operatorname{Lin}^{*} \sigma^{*} P_{n} \rightarrow \operatorname{BAff}\left(\mathbb{R}^{n} / \mathbb{Z}^{n}\right)
$$

is classified by the (conjugacy class of the) homomorphism

$$
\sigma_{*} \circ \operatorname{Lin}_{*}: \pi_{1}\left(\operatorname{BAff}\left(\mathbb{R}^{n} / \mathbb{Z}^{n}\right)\right) \rightarrow \pi_{1}\left(\operatorname{BAff}\left(\mathbb{R}^{n} / \mathbb{Z}^{n}\right)\right),
$$

which is the identity, as the composition of homomorphisms

$$
\sigma \circ \operatorname{Lin}: \operatorname{Aff}\left(\mathbb{R}^{n} / \mathbb{Z}^{n}\right) \rightarrow \operatorname{Aff}\left(\mathbb{R}^{n} / \mathbb{Z}^{n}\right)
$$

preserves path-connected components. Hence, the following cohomology rings are isomorphic

$$
\mathrm{H}^{*}\left(\operatorname{BAff}\left(\mathbb{R}^{n} / \mathbb{Z}^{n}\right) ; \mathbb{Z}_{\mathrm{id}_{*}}^{n}\right) \cong \mathrm{H}^{*}\left(\operatorname{BAff}\left(\mathbb{R}^{n} / \mathbb{Z}^{n}\right) ; \mathrm{H}_{1}\left(\mathbb{R}^{n} / \mathbb{Z}^{n} ; \mathbb{Z}\right)_{(\sigma \circ \mathrm{Lin})_{*}}\right) .
$$

There is a twisted version of the Leray-Serre spectral sequence, constructed by Siegel in Sie67, which allows to calculate the cohomology ring

$$
\mathrm{H}^{*}\left(\operatorname{BAff}\left(\mathbb{R}^{n} / \mathbb{Z}^{n}\right) ; \mathrm{H}_{1}\left(\mathbb{R}^{n} / \mathbb{Z}^{n} ; \mathbb{Z}\right)_{(\sigma \circ \operatorname{Lin})_{*}}\right)
$$


using the fibration

$$
\mathrm{B} \mathbb{R}^{n} / \mathbb{Z}^{n \hookrightarrow} \stackrel{\tau}{\longrightarrow} \mathrm{BAff}\left(\mathbb{R}^{n} / \mathbb{Z}^{n}\right) \stackrel{\operatorname{Lin}}{\longrightarrow} \mathrm{BGL}(n ; \mathbb{Z})
$$

and the system of local coefficients on $\operatorname{BGL}(n ; \mathbb{Z})$ of equation (29). Let $\breve{\mathrm{E}}_{2}^{p, q}$ denote the $\mathrm{E}_{2}$-page of this spectral sequence; since $\mathrm{BR}^{n} / \mathbb{Z}^{n}$ is simply connected, there is an exact sequence

$$
0 \longrightarrow \breve{\mathrm{E}}_{2}^{2,0} \stackrel{\operatorname{Lin}^{*}}{\longrightarrow} \mathrm{H}^{2}\left(\mathrm{BAff}\left(\mathbb{R}^{n} / \mathbb{Z}^{n}\right) ; \mathbb{Z}_{\mathrm{id}_{*}}\right) \stackrel{\tau^{*}}{\longrightarrow} \breve{\mathrm{E}}_{2}^{0,2},
$$

where the isomorphism of equation (30) is used tacitly. There are isomorphisms

$$
\begin{aligned}
& \breve{\mathrm{E}}_{2}^{2,0} \cong \mathrm{H}^{2}\left(\mathrm{BGL}(n ; \mathbb{Z}) ; \mathbb{Z}_{\mathrm{id}_{*}^{G}}^{n}\right) \\
& \breve{\mathrm{E}}_{2}^{0,2} \cong\left[\mathrm{H}^{2}\left(\mathrm{BR}^{n} / \mathbb{Z}^{n} ; \mathbb{Z}^{n}\right)\right]^{G} \subset \mathrm{H}^{2}\left(\mathrm{BR}^{n} / \mathbb{Z}^{n} ; \mathbb{Z}^{n}\right),
\end{aligned}
$$

where $\operatorname{id}_{*}^{G}: \pi_{1}(\operatorname{BGL}(n ; \mathbb{Z})) \cong \operatorname{GL}(n ; \mathbb{Z}) \rightarrow \operatorname{Aut}\left(\mathbb{Z}^{n}\right) \cong \mathrm{GL}(n ; \mathbb{Z})$ is the identity and $[.]^{G}$ denotes the group of $\operatorname{GL}(n ; \mathbb{Z})$-invariant elements. Since $\operatorname{ker} \tau^{*}=\operatorname{im~Lin}{ }^{*}$, it follows that $\psi^{-1}(\mu) \in \operatorname{im} p^{*}$. This completes the proof of Step 1.

Step 2. Consider the bundle of equation (28). This bundle admits a section, which is induced by the identity $\operatorname{map} \mathrm{BGL}(n ; \mathbb{Z}) \rightarrow \operatorname{BGL}(n ; \mathbb{Z})$. Thus

$$
\sigma^{*}\left(c_{U}\right)=0 \in \mathrm{H}^{2}\left(\operatorname{BGL}(n ; \mathbb{Z}) ; \mathbb{Z}_{\mathrm{id}_{*}^{G}}^{n}\right) .
$$

Moreover, if $\hat{\mathrm{E}}_{2, G}^{*, *}$ denotes the Leray-Serre spectral sequence for

$$
\mathbb{R}^{n} / \mathbb{Z}^{n} \hookrightarrow \sigma^{*} \operatorname{BGL}(n ; \mathbb{Z}) \rightarrow \operatorname{BGL}(n ; \mathbb{Z})
$$

with $\mathrm{H}_{1}\left(\mathbb{R}^{n} / \mathbb{Z}^{n} ; \mathbb{Z}\right)$ coefficients, the differential

$$
\hat{\mathrm{d}}_{G}^{(2)}: \hat{\mathrm{E}}_{2, G}^{0,1} \rightarrow \hat{\mathrm{E}}_{2, G}^{2,0}
$$

vanishes identically, as the bundle admits a section. Thus there is the following commutative diagram arising from functoriality of spectral sequences

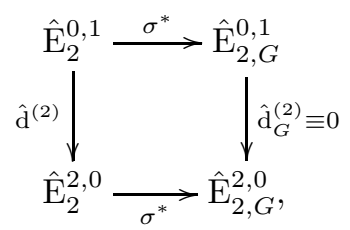

which implies that

$$
\sigma^{*} \hat{\mathrm{d}}^{(2)}\left(f^{1}\right)=0 .
$$

If $\psi_{G}: \mathrm{H}^{2}\left(\mathrm{BGL}(n ; \mathbb{Z}) ; \mathbb{Z}_{\mathrm{id}_{*}^{G}}^{n}\right) \rightarrow \hat{\mathrm{E}}_{2, G}^{2,0}$ is the isomorphism arising from the identification

$$
\mathrm{H}^{0}\left(\mathbb{R}^{n} / \mathbb{Z}^{n} ; \mathrm{H}_{1}\left(\mathbb{R}^{n} / \mathbb{Z}^{n} ; \mathbb{Z}\right)\right) \cong \mathbb{Z}^{n}
$$

there is a commutative diagram (cf. equation (25))

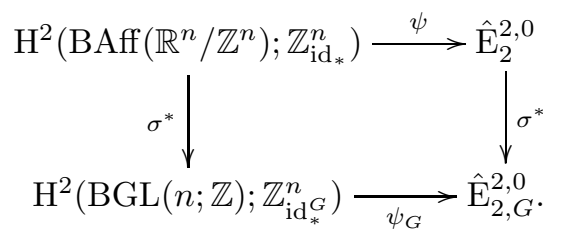

Equation (31) and the diagram (33) imply that

$$
\sigma^{*} \psi^{*}\left(c_{U}\right)=0 .
$$

Applying $\sigma^{*}$ to both sides of equation (27), and using equations (32) and (34), obtain that

$$
\sigma^{*} \mu=0,
$$


which proves Step 2.

By Step 1, there exists

such that $\psi^{-1}(\mu)=\operatorname{Lin}^{*} \nu$, then

$$
\nu \in \mathrm{H}^{2}\left(\mathrm{BGL}(n ; \mathbb{Z}) ; \mathbb{Z}_{\mathrm{id}_{*}^{G}}^{n}\right)
$$

$$
\sigma^{*} \circ \psi \circ \operatorname{Lin}^{*}(\nu)=\sigma^{*} \mu=0,
$$

since $\mu \in \operatorname{ker} \sigma^{*}$ by Step 2 . Commutativity of the diagram in equation (33) implies that

$$
\sigma^{*} \circ \psi \circ \operatorname{Lin}^{*}(\nu)=\psi_{G} \circ \sigma^{*} \circ \operatorname{Lin}^{*}(\nu) .
$$

Since $\psi_{G}$ is an isomorphism, equation (35) implies that

$$
\sigma^{*} \circ \operatorname{Lin}^{*}(\nu)=0
$$

as $\sigma^{*} \circ \operatorname{Lin}^{*}$ is the identity on $\mathrm{H}^{2}\left(\operatorname{BGL}(n ; \mathbb{Z}) ; \mathbb{Z}_{\mathrm{id}_{*}^{G}}^{n}\right)$, it follows that $\nu=0$. Therefore,

$$
\mu=0
$$

as required.

4.3. Relation to almost Lagrangian bundles. Throughout this section, fix an integral affine manifold $(B, \mathcal{A})$ with linear holonomy $\mathfrak{l}$ and an almost Lagrangian bundle $\mathbb{R}^{n} / \mathbb{Z}^{n} \hookrightarrow M \rightarrow B$ with Chern class

$$
c \in \mathrm{H}^{2}\left(B ; \mathbb{Z}_{\mathfrak{l}^{-T}}^{n}\right) .
$$

The aim of this section is to use Theorem 4.6 to compute the obstruction for the above bundle to be Lagrangian; Theorem 4.9 proves that this obstruction is given by the cohomology class of the cup product of the Chern class $c$ with the cohomology class of the symplectic form on the total space of the symplectic reference bundle associated to $(B, \mathcal{A})$ (cf. Lemma 4.7).

Symplectic reference bundles. The symplectic form $\omega_{0}$ on the total space of the symplectic reference bundle

$$
\mathbb{R}^{n} / \mathbb{Z}^{n} \hookrightarrow\left(\mathrm{T}^{*} B / P_{(B, \mathcal{A})}, \omega_{0}\right) \rightarrow B
$$

defines a cohomological invariant of $(B, \mathcal{A})$.

Lemma 4.7. The 2-form $\omega_{0}$ defines a cohomology class

$$
w_{0} \in \mathrm{H}^{1}\left(B ; \mathrm{H}^{1}\left(\mathbb{R}^{n} / \mathbb{Z}^{n} ; \mathbb{R}\right)_{\mathfrak{l}}\right),
$$

where $\mathfrak{l}: \pi_{1}(B) \rightarrow \mathrm{GL}(n ; \mathbb{Z}) \subset \mathrm{GL}(n ; \mathbb{R})$.

Proof. The cohomology theory used throughout this proof is Čech-de Rham. The cohomology class of a closed differential form can be represented as the obstruction to finding a globally defined potential. Let $\mathcal{U}=\left\{U_{\alpha}\right\}$ be a good open cover by integral affine coordinate neighbourhoods of $(B, \mathcal{A})$. There exist local action-angle coordinates $\left(\mathbf{a}_{\alpha}, \boldsymbol{\theta}_{\alpha}\right)$ on $\pi^{-1}\left(U_{\alpha}\right) \cong U_{\alpha} \times \mathbb{R}^{n} / \mathbb{Z}^{n}$, so that

$$
\omega_{\alpha}=\left.\omega_{0}\right|_{\pi^{-1}\left(U_{\alpha}\right)}=\sum_{i} \mathrm{~d} a_{\alpha}^{i} \wedge \mathrm{d} \theta_{\alpha}^{i}=\mathrm{d}\left(\sum_{i} a_{\alpha}^{i} \mathrm{~d} \theta_{\alpha}^{i}\right),
$$

The transition functions $\varphi_{\beta \alpha}$ for this choice of local trivialisations of $\mathrm{T}^{*} B / P_{(B, \mathcal{A})} \rightarrow B$ are given by

$$
\varphi_{\beta \alpha}\left(\mathbf{a}_{\alpha}, \boldsymbol{\theta}_{\alpha}\right)=\left(A_{\beta \alpha} \mathbf{a}_{\alpha}+\mathbf{d}_{\beta \alpha}, A_{\beta \alpha}^{-T} \boldsymbol{\theta}_{\alpha}\right),
$$

where $A_{\beta \alpha} \in \mathrm{GL}(n ; \mathbb{Z})$ and $\mathbf{d}_{\beta \alpha} \in \mathbb{R}^{n}$ is constant. For each $\alpha$, set

$$
\nu_{\alpha}=\sum_{i} a_{\alpha}^{i} \mathrm{~d} \theta_{\alpha}^{i}
$$

The cohomology class of $\omega_{0}$ (as a differential form on $\mathrm{T}^{*} B / P_{(B, \mathcal{A})}$ ) is given in Cech cohomology by the cocycle

$$
\tau_{\beta \alpha}=\varphi_{\beta \alpha}^{*} \nu_{\beta}-\nu_{\alpha}=\sum_{i, k} d_{\beta \alpha}^{i} \mathrm{~d} \theta_{\beta}^{i}
$$


Since $\mathcal{U}$ is a good cover for $B, \tau=\left\{\tau_{\beta \alpha}\right\}$ defines a one dimensional cohomology class $w_{0}$ on $B$ with coefficients in the local coefficient system

$$
\mathrm{H}^{1}\left(\mathbb{R}^{n} / \mathbb{Z}^{n} ; \mathbb{R}\right) \hookrightarrow P^{*} \rightarrow B
$$

whose monodromy is given by $\mathfrak{l}: \pi_{1}(B) \rightarrow \mathrm{GL}(n ; \mathbb{Z}) \subset \mathrm{GL}(n ; \mathbb{R})$, since this equals the inverse transposed of the monodromy of the period lattice bundle $P_{(B, \mathcal{A})}$. This proves the result.

Remark 4.2. If $\omega^{\prime}$ is any other symplectic form on $\mathrm{T}^{*} B / P_{(B, \mathcal{A})}$ making the topological reference bundle $\mathbb{R}^{n} / \mathbb{Z}^{n} \hookrightarrow \mathrm{T}^{*} B / P_{(B, \mathcal{A})} \rightarrow B$ Lagrangian, then there exists a 2 -form $\mu$ on $B$ such that

$$
\left(\mathrm{T}^{*} B / P_{(B, \mathcal{A})}, \omega^{\prime}\right) \quad \text { and } \quad\left(\mathrm{T}^{*} B / P_{(B, \mathcal{A})}, \omega_{0}+\pi^{*} \mu\right) \text {. }
$$

are fibrewise symplectomorphic. In particular, $\mu=s^{*} \omega^{\prime}$, where $s: B \rightarrow \mathrm{T}^{*} B / P_{(B, \mathcal{A})}$ is any section. Therefore $\omega^{\prime}-\pi^{*} s^{*} \omega^{\prime}$ also defines the cohomology class $w_{0}$, and the construction is independent of the choice of section $s$.

The cohomology class $w_{0}$ does not depend solely on the linear holonomy $\mathfrak{l}$ of $(B, \mathcal{A})$, as the next example illustrates (cf. Example 3.11).

Example 4.3. Let $\mathbb{R} / \mathbb{Z}$ and $\mathbb{R} / 2 \mathbb{Z}$ be the integral affine manifolds of Example 3.11 These affine manifolds have trivial linear holonomy. Let $\omega_{1}$ and $\omega_{2}$ be symplectic forms on their respective symplectic reference bundles. These bundles are isomorphic as affine $\mathbb{R} / \mathbb{Z}$-bundles and their total spaces can be identified with $S^{1} \times S^{1}$. The cohomology classes

$$
w_{0,1}, w_{0,2} \in \mathrm{H}^{1}\left(S^{1} ; \mathrm{H}^{1}(\mathbb{R} / \mathbb{Z} ; \mathbb{R})\right) \hookrightarrow \mathrm{H}^{2}\left(S^{1} \times S^{1} ; \mathbb{R}\right)
$$

defined from $\omega_{1}$ and $\omega_{2}$ as in Lemma 4.7 satisfy

$$
w_{0,2}=2 w_{0,1} .
$$

In particular, the above example hints at the fact that $w_{0}$ is an integral affine invariant of the manifold $(B, \mathcal{A})$. This is evident from the cocycle $\tau=\left\{\tau_{\beta \alpha}\right\}$ representing $w_{0}$ in the proof of Lemma 4.7, since it depends on the translational components of the changes of integral affine coordinates of $(B, \mathcal{A})$.

Remark 4.4. It is important to notice that the differential 1 -forms $\mathrm{d} \theta_{\alpha}^{1}, \ldots, \mathrm{d} \theta_{\alpha}^{n}$ represent, in fact, integral cohomology classes in

$$
\mathrm{H}^{1}\left(\mathbb{R}^{n} / \mathbb{Z}^{n} ; \mathbb{R}\right) \cong \mathrm{H}^{1}\left(\mathbb{R}^{n} / \mathbb{Z}^{n} ; \mathbb{Z}\right) \otimes_{\mathbb{Z}} \mathbb{R}
$$

for all indices $\alpha$. This is because these forms are dual to the flows of the vector fields $\partial / \partial \theta_{\alpha}^{1}, \ldots, \partial / \partial \theta_{\alpha}^{n}$ from time 0 to time 1 . These curves define a basis of the integral homology groups

$$
\mathrm{H}_{1}\left(\mathbb{R}^{n} / \mathbb{Z}^{n} ; \mathbb{Z}\right)
$$

of the fibres. Thus the reason why real coefficients are used throughout is that the translational components of the changes of integral affine coordinates of $(B, \mathcal{A})$ are not necessarily integral (cf. Remark 5.10).

Realisability theorem. Let $\mathrm{E}_{2, B}^{*, *}$ denote the $\mathrm{E}_{2}$-page of the Leray-Serre spectral sequence with integer coefficients of the almost Lagrangian bundle

$$
\mathbb{R}^{n} / \mathbb{Z}^{n} \hookrightarrow M \rightarrow B
$$

fixed above. Theorem 4.6 and naturality of the Leray-Serre spectral sequence imply the following corollary.

Corollary 4.8. Let $x \in \mathrm{E}_{2, B}^{p, 1}$. Its image under the differential $\mathrm{d}_{B}^{(2)}$ is given by

$$
\mathrm{d}_{B}^{(2)}(x)=(-1)^{p+1} \theta_{B}(x) \cdot{ }_{B} \psi_{B}(c),
$$

where $\theta_{B}, \psi_{B}, \cdot{ }_{B}$ are the pull-backs of $\theta, \psi, \cdot$ defined for the universal Lagrangian bundle.

The main idea of Theorem 4.9 is to study the differential

$$
\mathrm{d}_{B, \mathbb{R}}^{(2)}: \mathrm{E}_{2, B, \mathbb{R}}^{1,1} \rightarrow \mathrm{E}_{2, B, \mathbb{R}}^{3,0}
$$

on the $\mathrm{E}_{2}$-page of the Leray-Serre spectral sequence with real coefficients (hence the subscript $\mathbb{R}$ ) associated to the above almost Lagrangian bundle. Corollary 4.8 can be used to show that if $x \in \mathrm{E}_{2, B, \mathbb{R}}^{p, 1}$, then

$$
\mathrm{d}_{B, \mathbb{R}}^{(2)}(x)=(-1)^{p+1} \theta_{B, \mathbb{R}}(x) \cdot{ }_{B, \mathbb{R}} \psi_{B, \mathbb{R}}\left(c^{\mathbb{R}}\right),
$$


where $c^{\mathbb{R}} \in \mathrm{H}^{2}\left(B ; \mathbb{R}_{\chi_{*}}^{n}\right)$ is the image of $c$ under the homomorphism

$$
\mathrm{H}^{2}\left(B ; \mathbb{Z}_{\mathfrak{l}^{-T}}^{n}\right) \rightarrow \mathrm{H}^{2}\left(B ; \mathbb{R}_{\mathfrak{l}^{-T}}^{n}\right)
$$

induced by the standard inclusion

$$
\mathbb{Z}^{n} \hookrightarrow \mathbb{R}^{n} \cong \mathbb{Z}^{n} \otimes_{\mathbb{Z}} \mathbb{R}
$$

and $\theta_{B, \mathbb{R}}, \psi_{B, \mathbb{R}}$ are the appropriate isomorphisms. Henceforth, the subscripts $B, \mathbb{R}$ are omitted in order to simplify notation.

First, note that

$$
w_{0} \in \mathrm{E}_{2}^{1,1}
$$

since, up to a choice of basepoints, $\mathfrak{l}$ equals the local coefficient system

$$
\rho_{1}: \pi_{1}(B) \rightarrow \operatorname{Aut}\left(\mathrm{H}^{1}\left(\mathbb{R}^{n} / \mathbb{Z}^{n} ; \mathbb{R}\right)\right)
$$

which defines

$$
\mathrm{E}_{2}^{1,1} \cong \mathrm{H}^{1}\left(B ; \mathrm{H}^{1}\left(\mathbb{R}^{n} / \mathbb{Z}^{n} ; \mathbb{R}\right)_{\rho_{1}}\right) .
$$

Secondly, using local action-angle coordinates, it is possible to give an explicit cocycle representing the form $w_{0}$ in Čech-de Rham cohomology. Let $\mathcal{U}=\left\{U_{\alpha}\right\}$ be a good open cover by integral affine coordinate neighbourhoods of $(B, \mathcal{A})$. Remark 3.14 shows that there exist local trivialisations

$$
\Upsilon_{\alpha}: \pi^{-1}\left(U_{\alpha}\right) \rightarrow \mathrm{T}^{*} U_{\alpha} /\left.P_{(B, \mathcal{A})}\right|_{U_{\alpha}}
$$

inducing action-angle coordinates $\left(\mathbf{a}_{\alpha}, \boldsymbol{\theta}_{\alpha}\right)$ on $\pi^{-1}\left(U_{\alpha}\right)$; the corresponding transition functions $\varphi_{\beta \alpha}$ are of the form

$$
\varphi_{\beta \alpha}\left(\mathbf{a}_{\alpha}, \boldsymbol{\theta}_{\alpha}\right)=\left(A_{\beta \alpha} \mathbf{a}_{\alpha}+\mathbf{d}_{\beta \alpha}, A_{\beta \alpha}^{-T} \boldsymbol{\theta}_{\alpha}+\mathbf{g}_{\beta \alpha}\left(\mathbf{a}_{\alpha}\right)\right),
$$

where the first component corresponds to a change in integral affine coordinates on $(B, \mathcal{A})$. The family of 1-forms

$$
\bar{\tau}_{\beta \alpha}=\sum_{i=1}^{n} d_{\beta \alpha}^{i} \mathrm{~d} \theta_{\beta}^{i}
$$

define an element in $\mathrm{H}^{1}\left(B ; \mathrm{H}^{1}\left(\mathbb{R}^{n} / \mathbb{Z}^{n} ; \mathbb{R}\right)_{\rho_{1}}\right)$, since the forms $\mathrm{d} \theta_{\beta}^{1}, \ldots, \mathrm{d} \theta_{\beta}^{n}$ are closed and the family of cohomology classes of $\bar{\tau}_{\beta \alpha}$ form a Čech cocycle. This last statement holds since

$$
(\delta \bar{\tau})_{\alpha_{1} \alpha_{2} \alpha_{3}}=\mathrm{d}\left(\sum_{i=1}^{n} d_{\alpha_{2} \alpha_{3}}^{i} g_{\alpha_{2} \alpha_{1}}^{i}\right)
$$

where $\delta$ denotes Čech differential. Since the angle coordinates $\boldsymbol{\theta}_{\alpha}$ are pulled back from angle coordinates on the symplectic reference bundle via the local trivialisations $\Upsilon_{\alpha}$, it follows that the cohomology class that $\left\{\bar{\tau}_{\beta \alpha}\right\}$ defines equals the cohomology class $w_{0}$, since for all indices $\alpha, \beta$

$$
\tau_{\beta \alpha}=\bar{\tau}_{\beta \alpha}
$$

(cf. Lemma 4.7).

With the above constructions in place, it is possible to prove the main theorem of this paper.

Theorem 4.9. Let $(B, \mathcal{A})$ denote an integral affine manifold with linear holonomy l. An almost Lagrangian bundle $\mathbb{R}^{n} / \mathbb{Z}^{n} \hookrightarrow M \rightarrow B$ over $(B, \mathcal{A})$ is a Lagrangian bundle if and only if

$$
\mathrm{d}^{(2)}\left(w_{0}\right)=0
$$

where $\mathrm{d}^{(2)}: \mathrm{E}_{2}^{1,1} \rightarrow \mathrm{E}_{2}^{3,0}$ is the differential on the $\mathrm{E}^{2}$-page of the Leray-Serre spectral sequence for $\mathbb{R}^{n} / \mathbb{Z}^{n} \hookrightarrow$ $M \rightarrow B$ with real coefficients. 
Proof. Čech-de Rham cohomology and the corresponding interpretation of the Leray-Serre spectral sequence with real coefficients are used throughout this proof. Firstly, suppose that the bundle is, in fact, Lagrangian. Let $\omega$ denote a symplectic form on $M$ making $\mathbb{R}^{n} / \mathbb{Z}^{n} \hookrightarrow M \rightarrow B$ Lagrangian. Theorem 3.1 and equation (44) imply that there exists a good open cover $\mathcal{U}=\left\{U_{\alpha}\right\}$ of $B$ with local action-angle coordinates $\left(\mathbf{a}_{\alpha}, \boldsymbol{\theta}_{\alpha}\right)$ on each $\pi^{-1}\left(U_{\alpha}\right)$ and transition functions

$$
\varphi_{\alpha_{2} \alpha_{1}}\left(\mathbf{a}_{\alpha_{1}}, \boldsymbol{\theta}_{\alpha_{1}}\right)=\left(A_{\alpha_{2} \alpha_{1}} \mathbf{a}_{\alpha_{1}}+\mathbf{d}_{\alpha_{2} \alpha_{1}}, A_{\alpha_{2} \alpha_{1}}^{-T} \boldsymbol{\theta}_{\alpha_{1}}+\mathbf{g}_{\alpha_{2} \alpha_{1}}\left(\mathbf{a}_{\alpha_{1}}\right)\right),
$$

with the functions $\mathbf{g}_{\alpha_{2} \alpha_{1}}$ constrained by

$$
\varphi_{\alpha_{2} \alpha_{1}}^{*} \omega_{\alpha_{2}}=\omega_{\alpha_{1}}
$$

where $\omega_{\alpha_{i}}$ denotes the restriction of $\omega$ to $\pi^{-1}\left(U_{\alpha_{i}}\right)$ for $i=1,2$. On each intersection, equation (39) is equivalent to

$$
\sum_{i, j=1}^{n} A_{\alpha_{2} \alpha_{1}}^{i j} \mathrm{~d} a_{\alpha_{1}}^{j} \wedge \mathrm{d} g_{\alpha_{2} \alpha_{1}}^{i}=0
$$

since each $U_{\alpha_{1}} \cap U_{\alpha_{2}}$ is simply-connected (in fact, contractible), the forms $\mathrm{d} a_{\alpha_{1}}^{i}$ are exact and so the above equation implies that

As in the proof of Lemma 4.7 let

$$
\mathrm{d}\left(\sum_{i, j=1}^{n} A_{\alpha_{2} \alpha_{1}}^{i j} a_{\alpha_{1}}^{j} \mathrm{~d} g_{\alpha_{2} \alpha_{1}}^{i}\right)=0 .
$$

$$
\sigma_{\alpha}=\sum_{i=1}^{n} a_{\alpha}^{i} \mathrm{~d} \theta_{\alpha}^{i}
$$

be a locally defined potential for $\omega$. Since equation (39) holds,

$$
\kappa_{\alpha_{2} \alpha_{1}}=\varphi_{\alpha_{2} \alpha_{1}}^{*} \sigma_{\alpha_{2}}-\sigma_{\alpha_{1}}
$$

is a closed 1-form. The Čech cocycle $\left\{\kappa_{\alpha_{2} \alpha_{1}}\right\}$ represents the cohomology class of $\omega$ in $\mathrm{H}^{2}(M ; \mathbb{R})$. Take

$$
\underbrace{\sum_{i, j=1}^{n} A_{\alpha_{2} \alpha_{1}}^{i j} a_{\alpha_{1}}^{j} \mathrm{~d} g_{\alpha_{2} \alpha_{1}}^{i}}_{:=\zeta_{\alpha_{2} \alpha_{1}}}+\underbrace{\sum_{i=1}^{n} d_{\alpha_{2} \alpha_{1}}^{i} \mathrm{~d} \theta_{\alpha_{2}}^{i}}_{=\tau_{\alpha_{2} \alpha_{1}}}
$$

as a representative of $\kappa_{\alpha_{2} \alpha_{1}}$. Note that $\left\{\tau_{\alpha_{2} \alpha_{1}}\right\}$ is a representative of the cohomology class $w_{0} \in \mathrm{E}_{2}^{1,1}$; if $\delta$ denotes the Čech-boundary map, then

$$
(\delta \tau)_{\alpha_{1} \alpha_{2} \alpha_{3}}=\mathrm{d}\left(\sum_{i=1}^{n} d_{\alpha_{2} \alpha_{3}}^{i} g_{\alpha_{2} \alpha_{1}}^{i}\right):=\mathrm{d} \eta_{\alpha_{1} \alpha_{2} \alpha_{3}} .
$$

The local potentials $\eta_{\alpha_{1} \alpha_{2} \alpha_{3}}$ are defined up to a choice of constants. The functions $\left\{-(\delta \eta)_{\alpha_{1} \alpha_{2} \alpha_{3} \alpha_{4}}\right\}$ are a Cech-de Rham cocycle whose corresponding cohomology class in $\mathrm{E}_{2}^{3,0}$ is $\mathrm{d}^{(2)}\left(w_{0}\right)$ since the cover $\mathcal{U}$ is good (cf. Section 9 in [BT99]).

The Čech boundary of $\left\{\zeta_{\alpha_{2} \alpha_{1}}\right\}$ is equal to

$$
(\delta \zeta)_{\alpha_{1} \alpha_{2} \alpha_{3}}=\mathrm{d}\left(\sum_{i=1}^{n}\left(d_{\alpha_{1} \alpha_{3}}^{i}-d_{\alpha_{1} \alpha_{2}}^{i}\right) g_{\alpha_{1} \alpha_{2}}^{i}\right):=\mathrm{d} \xi_{\alpha_{1} \alpha_{2} \alpha_{3}} .
$$

A simple calculation shows that for all indices $\alpha_{1}, \alpha_{2}, \alpha_{3}, \alpha_{4}$

$$
(\delta \xi)_{\alpha_{1} \alpha_{2} \alpha_{3} \alpha_{4}}=-(\delta \eta)_{\alpha_{1} \alpha_{2} \alpha_{3} \alpha_{4}} .
$$

In particular, $\delta \xi$ is a Čech-de Rham cocycle representing the cohomology class $\mathrm{d}^{(2)}\left(w_{0}\right)$. In order to prove that this class vanishes, it suffices to show that $\xi_{\alpha_{1} \alpha_{2} \alpha_{3}}$ can be chosen so that

$$
(\delta \xi)_{\alpha_{1} \alpha_{2} \alpha_{3} \alpha_{4}}=0
$$


for all indices $\alpha_{1}, \alpha_{2}, \alpha_{3}, \alpha_{4}$.

Since equation (40) holds and the cover $\mathcal{U}$ is good, the 1 -forms $\zeta_{\alpha_{2} \alpha_{1}}$ are closed and, hence, exact. Set

$$
\zeta_{\alpha_{2} \alpha_{1}}=\mathrm{d} \epsilon_{\alpha_{2} \alpha_{1}}
$$

for each pair of indices $\alpha_{1}, \alpha_{2}$. Then

$$
(\delta \zeta)_{\alpha_{1} \alpha_{2} \alpha_{3}}=\mathrm{d}\left(\epsilon_{\alpha_{2} \alpha_{3}}-\epsilon_{\alpha_{1} \alpha_{3}}+\epsilon_{\alpha_{3} \alpha_{2}}\right)=\mathrm{d}(\delta \epsilon)_{\alpha_{1} \alpha_{2} \alpha_{3}} .
$$

Equations (41) and (43) imply that

$$
\xi_{\alpha_{1} \alpha_{2} \alpha_{3}}=(\delta \epsilon)_{\alpha_{1} \alpha_{2} \alpha_{3}}+C_{\alpha_{1} \alpha_{2} \alpha_{3}}
$$

for some constants $C_{\alpha_{1} \alpha_{2} \alpha_{3}}$. By substituting $\xi_{\alpha_{1} \alpha_{2} \alpha_{3}}$ with $\xi_{\alpha_{1} \alpha_{2} \alpha_{3}}-C_{\alpha_{1} \alpha_{2} \alpha_{3}}$, it may be assumed that

$$
\xi_{\alpha_{1} \alpha_{2} \alpha_{1}}=(\delta \epsilon)_{\alpha_{1} \alpha_{2} \alpha_{3}}
$$

which, in turn, implies that for all indices $\alpha_{1}, \alpha_{2}, \alpha_{3}, \alpha_{4}$

$$
(\delta \xi)_{\alpha_{1} \alpha_{2} \alpha_{3} \alpha_{4}}=\left(\delta^{2} \epsilon\right)_{\alpha_{1} \alpha_{2} \alpha_{3} \alpha_{4}}=0 .
$$

This proves that $\mathrm{d}^{(2)}\left(w_{0}\right)=0$.

Conversely, suppose that $\mathrm{d}^{(2)}\left(w_{0}\right)=0$ for the almost Lagrangian bundle $\mathbb{R}^{n} / \mathbb{Z}^{n} \hookrightarrow M \rightarrow B$. Let $\mathcal{U}=\left\{U_{\alpha}\right\}$ be the good cover of $B$ given by Remark 3.14 i.e. there exist local action-angle coordinates $\left(\mathbf{a}_{\alpha}, \boldsymbol{\theta}_{\alpha}\right)$ on $\pi^{-1}\left(U_{\alpha}\right)$ and the transition functions are of the form

$$
\varphi_{\alpha_{2} \alpha_{1}}\left(\mathbf{a}_{\alpha_{1}}, \boldsymbol{\theta}_{\alpha_{1}}\right)=\left(A_{\alpha_{2} \alpha_{1}} \mathbf{a}_{\alpha_{1}}+\mathbf{d}_{\alpha_{2} \alpha_{1}}, A_{\alpha_{2} \alpha_{1}}^{-T} \boldsymbol{\theta}_{\alpha_{1}}+\mathbf{g}_{\alpha_{2} \alpha_{1}}\left(\mathbf{a}_{\alpha_{1}}\right)\right),
$$

without the constraint on the functions $\mathbf{g}_{\alpha_{2} \alpha_{1}}$ given by equation (39). Recall that the form

$$
\omega_{\alpha}=\sum_{i=1}^{n} \mathrm{~d} a_{\alpha}^{i} \wedge \mathrm{d} \theta_{\alpha}^{i}
$$

makes the bundle $\mathbb{R}^{n} / \mathbb{Z}^{n} \hookrightarrow \pi^{-1}\left(U_{\alpha}\right) \rightarrow U_{\alpha}$ Lagrangian. The obstruction to patching these forms together to yield a globally defined symplectic form $\omega$ on $M$ which makes the bundle $\mathbb{R}^{n} / \mathbb{Z}^{n} \hookrightarrow M \rightarrow B$ Lagrangian is given by the Čech cocycle

$$
\varphi_{\alpha_{2} \alpha_{1}}^{*} \omega_{\alpha_{2}}-\omega_{\alpha_{1}}=\sum_{i, j=1}^{n} A_{\alpha_{2} \alpha_{1}}^{i j} \mathrm{~d} a_{\alpha_{1}}^{j} \wedge \mathrm{d} g_{\alpha_{2} \alpha_{1}}^{i}
$$

Since the cover $\mathcal{U}$ is good, this cocycle represents a cohomology class in $\mathrm{H}^{1}\left(B ; \mathcal{Z}^{2}\left(\mathrm{~T}^{*} B\right)\right)$, where $\mathcal{Z}^{2}\left(\mathrm{~T}^{*} B\right)$ denotes the sheaf of closed sections of the bundle $\Lambda^{2} \mathrm{~T}^{*} B \rightarrow B$. In light of the isomorphism

$$
\mathrm{H}^{1}\left(B ; \mathcal{Z}^{2}\left(\mathrm{~T}^{*} B\right)\right) \cong \mathrm{H}^{3}(B ; \mathbb{R})
$$

(cf. Theorem 8.1 in [BT99], [DD87]), the above cocycle defines a cohomology class

$$
v \in \mathrm{H}^{3}(B ; \mathbb{R}) \text {. }
$$

Using the notation of the first half of the proof, a cocycle representing $v$ in Cech cohomology is given by $-\delta \xi$ (this simply unravels the isomorphism of equation (45)). The equality of equation (42) still holds, since it is not necessary to have that the transition functions $\varphi_{\alpha_{2} \alpha_{1}}$ are symplectomorphisms in order to prove it. Thus

$$
-\delta \xi=\delta \eta
$$

Note that the Čech-de Rham cocycle $-\delta \eta$ is a representative of $\mathrm{d}^{(2)}\left(w_{0}\right)$; by assumption, this vanishes and

$$
v=0 .
$$

Therefore

$$
\sum_{i, j=1}^{n} A_{\alpha_{2} \alpha_{1}}^{i j} \mathrm{~d} a_{\alpha_{1}}^{j} \wedge \mathrm{d} g_{\alpha_{2} \alpha_{1}}^{i}
$$


is a coboundary; thus there exists closed 2-forms $k_{\alpha}$ defined on each $U_{\alpha}$ such that

$$
(\delta k)_{\alpha_{2} \alpha_{1}}=\sum_{i, j=1}^{n} A_{\alpha_{2} \alpha_{1}}^{i j} \mathrm{~d} a_{\alpha_{1}}^{j} \wedge \mathrm{d} g_{\alpha_{2} \alpha_{1}}^{i}
$$

for all indices $\alpha_{1}, \alpha_{2}$.

The forms

$$
\omega_{\alpha}-\pi^{*} k_{\alpha}
$$

defined on each $\pi^{-1}\left(U_{\alpha}\right)$ patch together by virtue of equations (44) and (46). Denote the resulting 2-form on $M$ by $\omega$. It is closed and non-degenerate since each $\omega_{\alpha}-\pi^{*} k_{\alpha}$ is; moreover, the fibres of the bundle are Lagrangian submanifolds of $(M, \omega)$, since they are Lagrangian submanifolds of the relevant symplectic manifold $\left(\pi^{-1}\left(U_{\alpha}\right), \omega_{\alpha}-\pi^{*} k_{\alpha}\right)$ and, hence, the result follows.

\section{Remark 4.5.}

i) Let $\mathbb{R}^{n} / \mathbb{Z}^{n} \hookrightarrow M \rightarrow B$ be an almost Lagrangian bundle over the integral affine manifold $(B, \mathcal{A})$ with Chern class $c$, and let $w_{0}$ be the cohomology class of Lemma 4.7. If

$$
\mathrm{d}^{(2)}: \mathrm{E}_{2}^{1,1} \rightarrow \mathrm{E}_{2}^{3,0}
$$

denotes the differential on the $E^{2}$-page of the Leray-Serre spectral sequence of $\mathbb{R}^{n} / \mathbb{Z}^{n} \hookrightarrow M \rightarrow B$ with real coefficients, Theorem 4.9 proves that

$$
\mathrm{d}^{(2)}\left(w_{0}\right)=-\mathcal{D}_{(B, \mathcal{A})}(c),
$$

where $\mathcal{D}_{(B, \mathcal{A})}$ denotes the homomorphism of Dazord and Delzant (cf. Theorem 3.3). This is because the cohomology class $\mathcal{D}_{(B, \mathcal{A})}(c)$ is given by the Čech-de Rham cocycle

$$
\left\{\sum_{i, j=1}^{n} A_{\beta \alpha}^{i j} \mathrm{~d} a_{\alpha}^{j} \wedge \mathrm{d} g_{\beta \alpha}^{i}\right\},
$$

whose cohomology class can also be represented by the Čech cocycle $-\delta \xi$ defined above. The relation of equation (42) proves the claim;

ii) Theorem 4.9 may be called a realisability theorem, since it provides a way to determine which cohomology classes in $\mathrm{H}^{2}\left(B ; \mathbb{Z}_{\mathfrak{l}-T}^{n}\right)$ can be realised as Chern classes of Lagrangian bundles over $(B, \mathcal{A})$. The terminology comes from the theory of symplectic realisations of Poisson manifolds (cf. Chapter 8 in [Vai94).

\section{Relation to Integral Affine Geometry}

5.1. The radiance obstruction of an affine manifold. In this section the radiance obstruction of an affine manifold is introduced, following the work of Goldman and Hirsch in GH84].

Universal radiance obstruction. Consider the group

$$
\operatorname{Aff}\left(\mathbb{R}^{n}\right)=\mathrm{GL}(n ; \mathbb{R}) \ltimes \mathbb{R}^{n} .
$$

and the associated standard exact sequence

$$
0 \longrightarrow \mathbb{R}^{n} \stackrel{\iota}{\longrightarrow} \mathrm{Aff}\left(\mathbb{R}^{n}\right) \stackrel{\operatorname{Lin}}{\longrightarrow} \mathrm{GL}(n ; \mathbb{R}) \longrightarrow 1 .
$$

Define

$$
\text { Trans }: \operatorname{Aff}\left(\mathbb{R}^{n}\right) \rightarrow \mathbb{R}^{n}
$$

$$
(A, \mathbf{b}) \mapsto \mathbf{b},
$$

which satisfies

$$
\operatorname{Trans}\left((A, \mathbf{b}) \cdot\left(A^{\prime}, \mathbf{b}^{\prime}\right)\right)=\operatorname{Trans}(A, \mathbf{b})+\operatorname{Lin}(A, \mathbf{b}) \operatorname{Trans}\left(A^{\prime}, \mathbf{b}^{\prime}\right),
$$

for all $(A, \mathbf{b}),\left(A^{\prime}, \mathbf{b}^{\prime}\right) \in \operatorname{Aff}\left(\mathbb{R}^{n}\right)$. Thus Trans is a crossed homomorphism and it defines an element

$$
r_{U} \in \mathrm{H}^{1}\left(\operatorname{Aff}\left(\mathbb{R}^{n}\right) ; \mathbb{R}_{\text {Lin }}^{n}\right),
$$

where $\mathbb{R}_{\text {Lin }}^{n}$ denotes $\mathbb{R}^{n}$ as an $\operatorname{Aff}\left(\mathbb{R}^{n}\right)$-module via the homomorphism Lin. 
Definition 5.1 (Universal radiance obstruction GH84, GH86]). The cohomology class $r_{U}$ is called the universal radiance obstruction.

Let $(B, \mathcal{A})$ be an affine manifold with affine holonomy representation

$$
\mathfrak{a}: \pi_{1}(B) \rightarrow \operatorname{Aff}\left(\mathbb{R}^{n}\right)
$$

(cf. Definition 3.9). Let $\mathfrak{l}=$ Lin $\circ \mathfrak{a}$ denote its linear holonomy and fix $\Gamma=\pi_{1}(B)$ throughout.

Definition 5.2 (Group theoretic definition of radiance obstruction GH84). The cohomology class

$$
r_{(B, \mathcal{A})}=\mathfrak{a}^{*} r_{U} \in \mathrm{H}^{1}\left(\Gamma ; \mathbb{R}_{\mathfrak{l}}^{n}\right)
$$

is called the radiance obstruction of the affine manifold $(B, \mathcal{A})$. An affine manifold $(B, \mathcal{A})$ whose radiance obstruction $r_{(B, \mathcal{A})}$ vanishes is called a radiant manifold.

Remark 5.3 (Characterisation of radiant manifolds GH84]). An affine manifold $(B, \mathcal{A})$ is radiant if and only if its affine holonomy representation $\mathfrak{a}$ can be chosen so that its image lies entirely in $\operatorname{GL}(n ; \mathbb{R}) \subset \operatorname{Aff}\left(\mathbb{R}^{n}\right)$. Equivalently, an affine manifold $(B, \mathcal{A})$ is radiant if and only if there exists an affine structure $\mathcal{A}^{\prime}$ which is affinely diffeomorphic to the given one and whose changes of coordinates lie in $\operatorname{GL}(n ; \mathbb{R})$.

The topology of the universal radiance obstruction. The universal radiance obstruction $r_{U}$ can also be described topologically as follows. Let $\mathfrak{a}: \Gamma \rightarrow \operatorname{Aff}\left(\mathbb{R}^{n}\right)$ be a representation of a discrete group $\Gamma$. The composition Trans $\circ \mathfrak{a}$ defines a crossed homomorphism which represents a cohomology class

$$
r_{\Gamma} \in \mathrm{H}^{1}\left(\Gamma ; \mathbb{R}_{\text {Linoa }}^{n}\right) \text {. }
$$

For any topological group $G$, let $G^{\delta}$ denote the group endowed with the discrete topology. In light of equation (47), there is a split short exact sequence

$$
0 \longrightarrow\left(\mathbb{R}^{n}\right)^{\delta} \stackrel{\iota}{\longrightarrow} \operatorname{Aff}\left(\mathbb{R}^{n}\right)^{\delta} \stackrel{\operatorname{Lin}}{\underset{\sigma}{\longleftrightarrow}} \mathrm{GL}(n ; \mathbb{R})^{\delta} \longrightarrow 1 .
$$

Applying Theorem 2.2 to the splitting $\sigma$, obtain a bundle

$$
\left(\mathbb{R}^{n}\right)^{\delta} \hookrightarrow \operatorname{BGL}(n ; \mathbb{R})^{\delta} \rightarrow \operatorname{BAff}\left(\mathbb{R}^{n}\right)^{\delta}
$$

where

$$
\operatorname{BGL}(n ; \mathbb{R})^{\delta} \simeq \operatorname{EAff}\left(\mathbb{R}^{n}\right)^{\delta} / \mathrm{GL}(n ; \mathbb{R})^{\delta} .
$$

Note that $\operatorname{BAff}\left(\mathbb{R}^{n}\right)^{\delta}$ and $\operatorname{BGL}(n ; \mathbb{R})^{\delta}$ are $\mathrm{K}\left(\operatorname{Aff}\left(\mathbb{R}^{n}\right) ; 1\right)$ and $\mathrm{K}(\mathrm{GL}(n ; \mathbb{R}) ; 1)$ respectively. Using the ideas of the proof of Theorem 2.4 the following lemma can be proved.

Lemma 5.1. There exists a bundle isomorphism

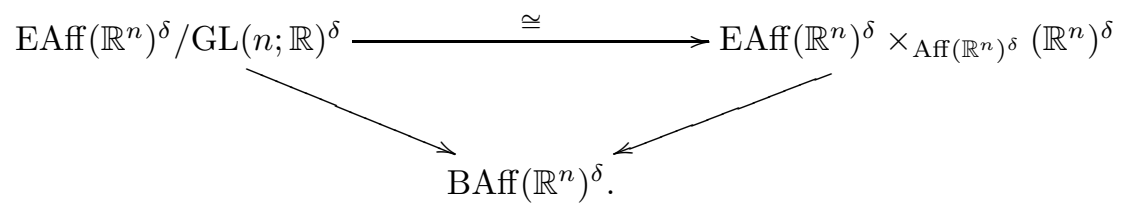

Let $\Gamma$ be a discrete topological group. Any representation

$$
\mathfrak{a}: \Gamma \rightarrow \operatorname{Aff}\left(\mathbb{R}^{n}\right)
$$

factors through $\operatorname{Aff}\left(\mathbb{R}^{n}\right)^{\delta}$; the homomorphism $\mathfrak{a}$ induces a map (defined up to homotopy)

$$
\overline{\mathfrak{a}}: \mathrm{B} \Gamma \rightarrow \mathrm{BAff}\left(\mathbb{R}^{n}\right)^{\delta}
$$

whose induced map on fundamental groups coincides with $\mathfrak{a}$ (up to a choice of basepoints). A lift

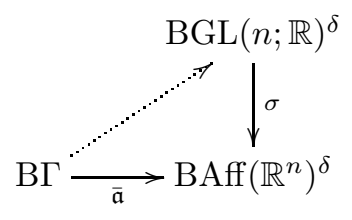


exists if and only if

$$
\overline{\mathfrak{a}}_{*}(\Gamma) \subset \sigma_{*}\left(\pi_{1}\left(\operatorname{BGL}(n ; \mathbb{R})^{\delta}\right)\right),
$$

since the fibre of the projection $\sigma: \operatorname{BGL}(n ; \mathbb{R})^{\delta} \rightarrow \operatorname{BAff}\left(\mathbb{R}^{n}\right)^{\delta}$ is discrete. Note that the induced map

$$
\sigma_{*}: \pi_{1}\left(\operatorname{BGL}(n ; \mathbb{R})^{\delta}\right) \cong \mathrm{GL}(n ; \mathbb{R}) \rightarrow \pi_{1}\left(\operatorname{BAff}\left(\mathbb{R}^{n}\right)^{\delta}\right) \cong \operatorname{Aff}\left(\mathbb{R}^{n}\right)
$$

equals the standard splitting

$$
\begin{aligned}
\sigma: \mathrm{GL}(n ; \mathbb{R}) & \rightarrow \operatorname{Aff}\left(\mathbb{R}^{n}\right) \\
A & \mapsto(A, \mathbf{0}) .
\end{aligned}
$$

by construction. Hence, the map $\overline{\mathfrak{a}}$ admits a lift if and only if the representation

$$
\overline{\mathfrak{a}}_{*}: \pi_{1}(\mathrm{~B} \Gamma) \cong \Gamma \rightarrow \pi_{1}\left(\mathrm{BAff}\left(\mathbb{R}^{n}\right)^{\delta}\right) \cong \operatorname{Aff}\left(\mathbb{R}^{n}\right)
$$

lies entirely within the image of $\sigma_{*}$. The latter statement is true if, up to conjugation, the image of the representation $\mathfrak{a}$ lies entirely in $\sigma(\mathrm{GL}(n ; \mathbb{R}))$, which is true if and only if $r_{\Gamma}=0$. In particular, letting $\Gamma=\operatorname{Aff}\left(\mathbb{R}^{n}\right)^{\delta}$ and $\mathfrak{a}: \operatorname{Aff}\left(\mathbb{R}^{n}\right)^{\delta} \rightarrow \operatorname{Aff}\left(\mathbb{R}^{n}\right)$ be the identity homomorphism, the above discussion proves the following theorem.

Theorem 5.2. The universal radiance obstruction $r_{U}$ is the obstruction to the existence of a section for the fibration

$$
\left(\mathbb{R}^{n}\right)^{\delta} \hookrightarrow \operatorname{BGL}(n ; \mathbb{R})^{\delta} \rightarrow \operatorname{BAff}\left(\mathbb{R}^{n}\right)^{\delta}
$$

A geometric interpretation. It is possible to give a geometric interpretation to the radiance obstruction of an affine manifold, which, thus far, is simply a cohomological invariant of its fundamental group. Firstly, note that the tangent bundle of an affine manifold can be endowed with the structure of a flat affine bundle.

Definition 5.4 (Flat affine bundle GH84]). Let $(F, \mathcal{B}) \hookrightarrow E \rightarrow N$ be an affine bundle, so that $(F, \mathcal{B})$ is an affine manifold and its structure group is $\operatorname{Aff}(F, \mathcal{B})$. It is said to be flat if it admits locally constant transition functions.

Example 5.5 (Tangent bundles of affine manifolds). The tangent bundle of an affine manifold $(N, \mathcal{A})$ (thought of as a vector bundle) is naturally a flat linear bundle, since the standard transition functions are locally constant. There is, however, a different choice of flat affine bundle structure that can be chosen on the tangent bundle of an affine manifold $(B, \mathcal{A})$, defined in GH84, which is more natural from the point of view of affinely flat geometry. The transition functions for the local affine trivialisations of this other flat affine bundle structure are simply given by the affine changes of coordinates on the base space (cf. [GH84]). This flat affine bundle is called the affine tangent bundle of the affine manifold $(B, \mathcal{A})$ and is denoted by $\mathrm{T}^{\text {Aff }} B \rightarrow B$.

Fix an $n$-dimensional manifold $(B, \mathcal{A})$ and let $\mathrm{T}^{\mathrm{Aff}} B \rightarrow B$ be its affine tangent bundle. Since its fibres are contractible, there exists a section to this bundle, e.g. the zero section. However, it is not necessarily true that the affine tangent bundle admits a flat section.

Definition 5.6 (Flat section of a flat affine bundle [GH84]). Let $(F, \mathcal{B}) \hookrightarrow E \rightarrow N$ be a flat affine bundle. A section $s: N \rightarrow E$ is flat if, for any local trivialisation $\pi^{-1}\left(U_{\alpha}\right) \rightarrow U_{\alpha} \times F$, the composite

$$
U_{\alpha} \stackrel{s_{\alpha}}{\longrightarrow} \pi^{-1}\left(U_{\alpha}\right) \longrightarrow U_{\alpha} \times F \stackrel{\mathrm{pr}_{2}}{\longrightarrow} F
$$

is locally constant.

Fix an affine manifold $(B, \mathcal{A})$ and let $\mathrm{T}^{\mathrm{Aff}} B \rightarrow B$ denote the corresponding affine tangent bundle with transition functions $f_{\beta \alpha}$. Since the transition functions of $\mathrm{T}^{\mathrm{Aff}} B \rightarrow B$ are constant on connected components, it is possible to construct the bundle

$$
\left(\mathbb{R}^{n}\right)^{\delta} \hookrightarrow\left(\mathrm{T}^{\mathrm{Aff}} B\right)^{\delta} \rightarrow B
$$

obtained by endowing the fibres with the discrete topology. Denote its classifying map by

$$
\chi_{\mathrm{Aff}}: B \rightarrow \operatorname{BAff}\left(\mathbb{R}^{n}\right)^{\delta} .
$$


In light of Lemma 5.1, the bundle of equation (49) is isomorphic to the pull-back

$$
\chi_{\mathrm{Aff}}^{*} \mathrm{BGL}(n ; \mathbb{R})^{\delta} \rightarrow B .
$$

Therefore the cohomology class

$$
\chi_{\mathrm{Aff}}^{*} r_{U}
$$

is the obstruction to the existence of a section for the bundle of equation (49).

The homotopy class of $\chi_{\mathrm{Aff}}$ is determined by its induced map on fundamental groups, since BAff $\left(\mathbb{R}^{n}\right)^{\delta}$ is a $\mathrm{K}\left(\operatorname{Aff}\left(\mathbb{R}^{n}\right) ; 1\right)$. By construction, this map equals (up to conjugation) the affine holonomy

$$
\mathfrak{a}: \pi_{1}(B)=\Gamma \rightarrow \operatorname{Aff}\left(\mathbb{R}^{n}\right)
$$

of the affine manifold $(B, \mathcal{A})$ (cf. [AM55). Let

$$
\overline{\mathfrak{a}}: \mathrm{B} \Gamma \rightarrow \operatorname{Aff}\left(\mathbb{R}^{n}\right)^{\delta}
$$

be the map (defined up to homotopy) induced by the affine holonomy and let $\chi_{\tilde{B}}: B \rightarrow \mathrm{B} \Gamma$ denote the classifying map for the universal covering $\tilde{B} \rightarrow B$. Then (up to homotopy)

$$
\chi_{\mathrm{Aff}}=\overline{\mathfrak{a}} \circ \chi_{\tilde{B}}
$$

in particular,

$$
\chi_{\mathrm{Aff}}^{*} r_{U}=\chi_{\tilde{B}}^{*} \circ \overline{\mathfrak{a}}^{*} r_{U}=\chi_{\tilde{B}}^{*} r_{(B, \mathcal{A})} .
$$

The map $\chi_{\tilde{B}}^{*}$ is an isomorphism on one dimensional cohomology with any coefficient system (cf. GH84]) and thus

$$
r_{(B, \mathcal{A})}=\left(\chi_{\tilde{B}}^{*}\right)^{-1} \circ \chi_{\mathrm{Aff}}^{*} r_{U} .
$$

Remark 5.7. By abuse of nomenclature and notation, the class $\chi_{\mathrm{Aff}}^{*} r_{U}$ is henceforth also referred to as the radiance obstruction of $(B, \mathcal{A})$ and denoted by $r_{(B, \mathcal{A})}$.

With this identification, the radiance obstruction $r_{(B, \mathcal{A})}$ is the obstruction to the existence of a section to the bundle of equation (49). By construction, a section for the aforementioned bundle exists if and only if $\mathrm{T}^{\mathrm{Aff}} B \rightarrow B$ admits a flat section. Therefore the following theorem holds.

Theorem 5.3 (Goldman and Hirsch [GH84]). Let $(B, \mathcal{A})$ be an n-dimensional affine manifold with linear holonomy $\mathfrak{l}$. The radiance obstruction

$$
r_{(B, \mathcal{A})} \in \mathrm{H}^{1}\left(B ; \mathbb{R}_{\mathrm{l}}^{n}\right)
$$

is the obstruction to the existence of a flat section for the flat affine bundle $\mathrm{T}^{\mathrm{Aff}} B \rightarrow B$.

Example 5.8 (Inequivalent flat affine structures on $\mathrm{T}\left(S^{1} \times \mathbb{R}\right)$ ).

i) Consider the $\mathbb{Z}$-action on $\mathbb{R}^{2}$ given by translations in a fixed direction $\mathbf{b}_{0} \neq 0$; this action is free, proper and by affine transformations on $\mathbb{R}^{2}$. Thus the manifold $\mathbb{R}^{2} / \mathbb{Z}$ is affine and its affine holonomy

$$
\mathfrak{a}_{\mathbb{R}^{2} / \mathbb{Z}}: \pi_{1}\left(\mathbb{R}^{2} / \mathbb{Z}\right) \rightarrow \operatorname{Aff}\left(\mathbb{R}^{2}\right)
$$

is defined on a generator $\gamma$ as

$$
\mathfrak{a}_{\mathbb{R}^{2} / \mathbb{Z}}(\gamma)=\left(I, \mathbf{b}_{0}\right)
$$

and extended by linearity. The crossed homomorphism Trans $\circ \mathfrak{a}_{\mathbb{R}^{2} / \mathbb{Z}}$ defines a non-zero cohomology class

$$
r_{\mathbb{R}^{2} / \mathbb{Z}} \in \mathrm{H}^{1}\left(\mathbb{R}^{2} / \mathbb{Z} ; \mathbb{R}^{2}\right)
$$

In light of Theorem 5.3, the flat affine bundle $\mathrm{T}^{\mathrm{Aff}}\left(\mathbb{R}^{2} / \mathbb{Z}\right) \rightarrow \mathbb{R}^{2} / \mathbb{Z}$ does not admit a flat section;

ii) The inclusion

$$
\mathbb{R}^{2} \backslash\{\mathbf{0}\} \hookrightarrow \mathbb{R}^{2}
$$

induces an affine structure on $\mathbb{R}^{2} \backslash\{\mathbf{0}\}$ which has trivial affine holonomy. Therefore the affine tangent bundle

$$
\mathrm{T}^{\mathrm{Aff}}\left(\mathbb{R}^{2} \backslash\{\mathbf{0}\}\right) \rightarrow \mathbb{R}^{2} \backslash\{\mathbf{0}\}
$$

admits a flat section. 
5.2. Relation to Lagrangian bundles. In this section, the radiance obstruction $r_{(B, \mathcal{A})}$ is related to the problem of constructing Lagrangian bundles over $B$ inducing the integral affine structure $\mathcal{A}$. Throughout the following, fix an integral affine manifold $(B, \mathcal{A})$ whose linear holonomy is denoted by $\mathfrak{l}$.

Recall that $w_{0}$ is the cohomology class of the symplectic form $\omega_{0}$ of the symplectic reference bundle associated to $(B, \mathcal{A})$

$$
\mathbb{R}^{n} / \mathbb{Z}^{n} \hookrightarrow\left(\mathrm{T}^{*} B / P_{(B, \mathcal{A})}, \omega_{0}\right) \rightarrow B
$$

Let $\mathcal{C}^{\infty}\left(\mathrm{T}^{\text {Aff }} B\right)$ and $\mathcal{C}^{\infty}\left(\mathrm{T}^{*} \mathbb{R}^{n} / \mathbb{Z}^{n}\right)$ denote the sheaves of sections of the affine tangent bundle $\mathrm{T}^{\text {Aff }} B \rightarrow B$ and of 1 -forms on the fibres of the symplectic reference bundle respectively. The symplectic form $\omega_{0}$ defines an isomorphism of sheaves

$$
\mathcal{C}^{\infty}\left(\mathrm{T}^{*} \mathbb{R}^{n} / \mathbb{Z}^{n}\right) \cong \mathcal{C}^{\infty}\left(\mathrm{T}^{\mathrm{Aff}} B\right)
$$

In local action-angle coordinates $\left(\mathbf{a}_{\alpha}, \boldsymbol{\theta}_{\alpha}\right)$, the above isomorphism is given by

$$
\sum_{i=1}^{n} h_{\alpha}^{i} \mathrm{~d} \theta_{\alpha}^{i} \mapsto \sum_{i=1}^{n} h_{\alpha}^{i} \frac{\partial}{\partial a_{\alpha}^{i}},
$$

where $h_{\alpha}^{1}, \ldots, h_{\alpha}^{n}$ are smooth functions. By equation (52), the isomorphism of equation (51) restricted to the subsheaf $\mathcal{C}_{\text {flat }}^{\infty}\left(\mathrm{T}^{*} \mathbb{R}^{n} / \mathbb{Z}^{n}\right)$ of locally constant sections descends to an isomorphism of sheaves

$$
\mathcal{C}_{\text {flat }}^{\infty}\left(\mathrm{T}^{*} \mathbb{R}^{n} / \mathbb{Z}^{n}\right) \cong \mathcal{C}_{\text {flat }}^{\infty}\left(\mathrm{T}^{\mathrm{Aff}} B\right) .
$$

Since $\mathrm{T}^{*} \mathbb{R}^{n} / \mathbb{Z}^{n}$ admits a frame of closed forms, a locally constant section is given in local action-angle coordinates by

$$
\sum_{i=1}^{n} r_{\alpha}^{i} \mathrm{~d} \theta_{\alpha}^{i}
$$

where $r_{\alpha}^{1}, \ldots, r_{\alpha}^{n} \in \mathbb{R}$ are constant. Such sections are in 1-1 correspondence with cohomology classes in $\mathrm{H}^{1}\left(\mathbb{R}^{n} / \mathbb{Z}^{n} ; \mathbb{R}\right)$, since the forms $\mathrm{d} \theta_{\alpha}^{1}, \ldots, \mathrm{d} \theta_{\alpha}^{n}$ induce a basis of $\mathrm{H}^{1}\left(\mathbb{R}^{n} / \mathbb{Z}^{n} ; \mathbb{R}\right)$. Hence, the symplectic form $\omega_{0}$ induces an isomorphism of sheaves

$$
\mathcal{P}^{*} \cong \mathcal{C}_{\text {flat }}^{\infty}\left(\mathrm{T}^{\mathrm{Aff}} B\right),
$$

where $\mathcal{P}^{*}$ denotes the sheaves of sections of the local coefficient system

$$
\mathrm{H}^{1}\left(\mathbb{R}^{n} / \mathbb{Z}^{n} ; \mathbb{R}\right) \hookrightarrow P^{*} \rightarrow B
$$

associated to the symplectic reference bundle. This isomorphism induces an isomorphism of cohomology groups

$$
\Phi: \mathrm{H}^{*}\left(B ; \mathcal{P}^{*}\right) \rightarrow \mathrm{H}^{*}\left(B ; \mathcal{C}_{\text {flat }}^{\infty}\left(\mathrm{T}^{\mathrm{Aff}} B\right)\right) .
$$

Since both $\mathcal{P}^{*}$ and $\mathcal{C}_{\text {flat }}^{\infty}\left(\mathrm{T}^{\mathrm{Aff}} B\right)$ are locally constant sheaves, the above induces an isomorphism

$$
\Phi: \mathrm{H}^{*}\left(B ; \mathrm{H}^{1}\left(\mathbb{R}^{n} / \mathbb{Z}^{n} ; \mathbb{R}\right)_{\mathfrak{l}}\right) \rightarrow \mathrm{H}^{1}\left(B ; \mathbb{R}_{\mathfrak{l}}^{n}\right) .
$$

Theorem 5.4. The cohomology class $w_{0} \in \mathrm{H}^{1}\left(B ; \mathrm{H}^{1}\left(\mathbb{R}^{n} / \mathbb{Z}^{n} ; \mathbb{R}\right)_{\mathfrak{l}}\right)$ defined by the symplectic form $\omega_{0}$ as in Lemma 4.7. maps to the radiance obstruction $r_{(B, \mathcal{A})} \in \mathrm{H}^{1}\left(B ; \mathbb{R}_{\mathfrak{l}}^{n}\right)$.

Proof. By Theorem [5.3, the radiance obstruction $r_{(B, \mathcal{A})}$ is the obstruction to the existence of a flat section to $\mathrm{T}^{\mathrm{Aff}} B \rightarrow B$. Let $\mathcal{U}=\left\{U_{\alpha}\right\}$ be a good open cover by integral affine coordinate neighbourhoods of $(B, \mathcal{A})$ and let

$$
\phi_{\alpha}: U_{\alpha} \rightarrow \mathbb{R}^{n}
$$

denote the coordinate map. The section

$$
\begin{aligned}
s_{\alpha}: U_{\alpha} & \rightarrow \mathrm{T}^{\mathrm{Aff}} U_{\alpha} \\
x & \mapsto\left(x,-\left(D \phi_{\alpha}(x)\right)^{-1}\left(\phi_{\alpha}(x)\right)\right)
\end{aligned}
$$

is flat (cf. GH84). The collection

$$
\hat{\tau}=\left\{\hat{\tau}_{\beta \alpha}\right\}:=\left\{s_{\beta}-s_{\alpha}\right\}
$$


is a Čech cocycle which represents $r_{(B, \mathcal{A})}$ (cf. GH84]).

Let $\mathbf{a}_{\alpha}$ denote affine coordinates on $U_{\alpha}$ induced by $\phi_{\alpha}$ and, as usual, set

$$
\phi_{\beta} \circ \phi_{\alpha}^{-1}\left(\mathbf{a}_{\alpha}\right)=A_{\beta \alpha} \mathbf{a}_{\alpha}+\mathbf{d}_{\beta \alpha} \in \operatorname{Aff}_{\mathbb{Z}}\left(\mathbb{R}^{n}\right) .
$$

The difference $s_{\beta}-s_{\alpha}$ is given by

$$
\hat{\tau}_{\beta \alpha}=\sum_{i=1}^{n} d_{\beta \alpha}^{i} \frac{\partial}{\partial a_{\beta}^{i}}
$$

In light of equation (52), the preimage of $\hat{\tau}_{\beta \alpha}$ under the isomorphism of equation (53) is given by

$$
\sum_{i=1}^{n} d_{\beta \alpha}^{i} \mathrm{~d} \theta_{\beta}^{i}
$$

The cocycle of equation (57) corresponds to the cocycle $\tau$ defining the cohomology class $w_{0}$ in the proof of Lemma 4.7 and, thus, the result follows.

Theorem 5.4 allows to use tools from affine geometry to study problems in the symplectic geometry of Lagrangian bundles and vice versa. For instance, the following theorem holds.

Theorem 5.5. There exist no closed radiant integral affine manifolds.

Proof. Suppose the contrary. Let $(B, \mathcal{A})$ be a closed radiant integral affine manifold and let $P_{(B, \mathcal{A})}$ denote the associated period lattice bundle (cf. Definition 3.8). Consider the symplectic reference bundle

$$
\mathbb{R}^{n} / \mathbb{Z}^{n} \hookrightarrow\left(\mathrm{T}^{*} B / P_{(B, \mathcal{A})}, \omega_{0}\right) \rightarrow B
$$

since $B$ and $\mathbb{R}^{n} / \mathbb{Z}^{n}$ are closed, so is $\mathrm{T}^{*} B / P_{(B, \mathcal{A})}$. Therefore the cohomology class

$$
\left[\omega_{0}\right] \in \mathrm{H}^{2}\left(\mathrm{~T}^{*} B / P_{(B, \mathcal{A})} ; \mathbb{R}\right)
$$

is non-zero. Lemma 4.7 proves that $\left[\omega_{0}\right]$ vanishes if and only if $w_{0}$ vanishes. However, Theorem $[5.4$ implies that

$$
w_{0}=\Phi^{-1}\left(r_{(B, \mathcal{A})}\right)=0,
$$

where the second equality follows by assumption. Therefore $\left[\omega_{0}\right]=0$, but this is a contradiction.

Remark 5.9. Theorem 5.5 can be used, for instance, to prove that, for $n \geq 2, S^{n}$ is not an integral affine manifold (cf. Example 2.2 and Lemma 2.2 in [Sep10b]). This is because $\pi_{1}\left(S^{n}\right)=\{0\}$, which implies that, were $S^{n}$ to be an integral affine manifold, its radiance obstruction would vanish, which contradicts Theorem 5.5. In fact, the argument of Example 2.2 in Sep10b proves directly that if $S^{3}$ were an integral affine manifold then its radiance obstruction would vanish by noticing that the cohomology of the total space of the associated symplectic reference bundle (which would necessarily be diffeomorphic to $S^{3} \times \mathbb{R}^{3} / \mathbb{Z}^{3}$ ) in degree 2 comes from the inclusion of the fibre $\mathbb{R}^{3} / \mathbb{Z}^{3} \hookrightarrow S^{3} \times \mathbb{R}^{3} / \mathbb{Z}^{3}$ (which induces an isomorphism in cohomology via the Künneth formula).

Remark 5.10. It is important to notice the difference between the bundle $P^{*} \rightarrow B$ and the period lattice bundle $P \rightarrow B$ associated to an integral affine manifold $(B, \mathcal{A})$. The former is an affine invariant of $B$ (via the symplectic form $\omega_{0}$ ), while the latter is only a linear invariant, since it is the pull-back of a universal lattice defined over $\operatorname{BGL}(n ; \mathbb{Z})$. The period lattice bundle can be endowed with the structure of an affine lattice of $(B, \mathcal{A})$ if and only if the radiance obstruction $r_{(B, \mathcal{A})}$ is an integral form, which, in turn, is true if and only if the coordinate changes of the atlas $\mathcal{A}$ can be chosen to lie in the group of affine transformations of $\mathbb{Z}^{n}$

$$
\operatorname{Aff}\left(\mathbb{Z}^{n}\right):=\mathrm{GL}(n ; \mathbb{Z}) \ltimes \mathbb{Z}^{n} .
$$

Such manifolds are henceforth called strongly integral affine manifolds, although it must be noticed that this terminology is not standard (cf. [GS06]). In view of Theorem [5.4, the symplectic form $\omega_{0}$ on the symplectic reference bundle of a strongly integral affine manifold $(B, \mathcal{A})$ is itself integral.

Combining Theorems 4.9 and [5.4, obtain the main result of the paper. 
Main Result. Let $(B, \mathcal{A})$ denote an integral affine manifold with linear holonomy $\mathfrak{l}$. An almost Lagrangian bundle $\mathbb{R}^{n} / \mathbb{Z}^{n} \hookrightarrow M \rightarrow B$ over $(B, \mathcal{A})$ is Lagrangian if and only if its Chern class $c \in \mathrm{H}^{2}\left(B ; \mathbb{Z}_{\mathfrak{l}^{-T}}^{n}\right)$ satisfies

$$
\theta_{B}\left(\Phi^{-1}\left(r_{(B, \mathcal{A})}\right)\right) \cdot \psi_{B}\left(c^{\mathbb{R}}\right)=0
$$

where the notation is the same as in Corollary 4.8, and $r_{(B, \mathcal{A})}$ is the radiance obstruction of the integral affine manifold $(B, \mathcal{A})$.

Remark 5.11. Using the fact that the radiance obstruction $r_{(B, \mathcal{A})}$ is the cohomology class defined by the identity map Id : T $B \rightarrow \mathrm{T} B$ (cf. [GH84]), the above result implies Theorem 3 in Sep11]. This can be seen as follows. The left hand side of equation (59) equals $\mathcal{D}_{(B, \mathcal{A})}(c)$ by Theorem 4.9] using Cech cocycles for $c$ and $r_{(B, \mathcal{A})}$, it can be checked that the pairing $\theta_{B}\left(\Phi^{-1}\left(r_{(B, \mathcal{A})}\right)\right) \cdot \psi_{B}\left(c^{\mathbb{R}}\right)$ yields a Čech cocycle whose corresponding cohomology class in $\mathrm{H}^{3}(B ; \mathbb{R})$ equals the twisted cup product of $c$ as defined in Sep11, thus yielding the result.

The Main Result proves that the homomorphism $\mathcal{D}_{(B, \mathcal{A})}$ of Dazord and Delzant [DD87] is completely determined by the integral affine structure on the base of an almost Lagrangian bundle and by the universal Chern class $c_{U}$.

Remark 5.12. If $(B, \mathcal{A})$ is a strongly integral affine manifold with linear holonomy $\mathfrak{l}$, the Main Result can be strengthened to say that an almost Lagrangian bundle over $(B, \mathcal{A})$ is Lagrangian if and only if its Chern class $c \in \mathrm{H}^{2}\left(B ; \mathbb{Z}_{\mathfrak{l}-T}^{n}\right)$ satisfies

$$
\theta\left(\Phi^{-1}\left(r_{(B, \mathcal{A})}\right)\right) \cdot \psi(c)=0 .
$$

In particular, if $\mathbb{R}^{n} / \mathbb{Z}^{n} \hookrightarrow(M, \omega) \rightarrow B$ is a Lagrangian bundle over a strongly integral affine manifold $(B, \mathcal{A})$ (i.e. it induces the affine structure $\mathcal{A}$ on $B$ ), then $\omega$ can always be chosen to be integral. This should be compared with Remark 1.2 of [GS06. Note that it is not true that for a fixed integral affine manifold $(B, \mathcal{A})$ there is a strongly integral affine manifold $\left(B^{\prime}, \mathcal{A}^{\prime}\right)$ in the same integral affine diffeomorphism class. This can be seen by considering an integral affine two-torus with trivial linear holonomy and translational components which are not integral (cf. Mis96).

The following corollary is a special case of the Main Result.

Corollary 5.6. If $(B, \mathcal{A})$ is a radiant affine manifold, then $\mathcal{D}_{(B, \mathcal{A})}=0$.

Remark 5.13. Corollary 5.6 should be compared with what is known in the literature regarding exactness of the symplectic form on the total space of a Lagrangian (or isotropic) bundle, e.g. [Dui80.

5.3. Some examples. In this section a manifold is endowed with various radiant integral affine structures to illustrate how the classification of Lagrangian bundles depends on the integral affine geometry of the base space.

The manifold $B=\mathbb{R}^{2} \backslash\{\mathbf{0}\}$ inherits an integral affine structure from $\mathbb{R}^{2}$ via the natural inclusion

$$
B \hookrightarrow \mathbb{R}^{2} .
$$

Denote this integral affine structure by $\mathcal{A}_{0}$. Its universal cover $\tilde{B}$ can also be endowed with an integral affine structure $\tilde{\mathcal{A}}_{0}$; an explicit description of the affine structure on $\tilde{B}$ can be found in [BF07]. It is important to notice that this affine structure on $\tilde{B}$ is not affinely isomorphic to the standard affine structure on $\mathbb{R}^{2}$. For any matrix $A \in \mathrm{GL}(2 ; \mathbb{Z})$, it is possible to define a $\mathbb{Z}$-action on $\left(\tilde{B}, \tilde{\mathcal{A}}_{0}\right)$ which induces an integral affine structure on $B$ whose affine holonomy is given by the representation defined on the generator $\gamma$ of $\pi_{1}(B)$ by

$$
\gamma \mapsto(A, \mathbf{0}) \text {. }
$$

For $A_{1}, A_{2}, A_{3} \in \mathrm{SL}(2 ; \mathbb{Z})$, let $\mathcal{A}_{1}, \mathcal{A}_{2}, \mathcal{A}_{3}$ be the corresponding radiant integral affine structures on $B$. Consider the integral affine manifold

$$
\left(Y, \mathcal{A}_{Y_{3}}\right)=\left(B, \mathcal{A}_{1}\right) \times\left(B, \mathcal{A}_{2}\right) \times\left(B, \mathcal{A}_{3}\right) .
$$

This affine manifold is radiant, as it can be seen by looking at its affine holonomy. Thus $\mathcal{D}_{\left(Y, \mathcal{A}_{Y}\right)}=0$ by Corollary 5.6. Note that $Y$ has the homotopy type of a three-torus and so it has $\mathrm{H}^{3}(Y ; \mathbb{R}) \cong \mathbb{R}$. This integral affine manifold therefore provides an example of trivial homomorphism $\mathcal{D}_{\left(Y, \mathcal{A}_{Y}\right)}$ of Dazord and Delzant even 
though its range is non-trivial.

Let $\mathfrak{l}_{Y}$ denote the linear holonomy of $\left(Y, \mathcal{A}_{Y}\right)$. The twisted cohomology group

$$
\mathrm{H}^{2}\left(Y ; \mathbb{Z}_{\mathfrak{l}_{Y}^{-T}}^{6}\right)
$$

is not trivial if and only if at least one of the $A_{i}$ is unipotent. If this condition is satisfied, then $\left(Y, \mathcal{A}_{Y}\right)$ provides the first example of a manifold whose associated homomorphism $\mathcal{D}_{\left(Y, \mathcal{A}_{Y}\right)}$ is trivial notwithstanding the fact that both its domain and range are not trivial. More generally, by taking the product of $k$ radiant integral affine manifolds of the form described above, it is possible to construct such examples in any even dimension greater than or equal to 6 .

Consider the product

$$
\left(Z_{\mathbf{n}}, \mathcal{A}_{Z_{\mathbf{n}}}\right)=\left(B, \mathcal{A}_{n_{1}}\right) \times \ldots\left(B, \mathcal{A}_{n_{k}}\right)
$$

where $\mathbf{n}=\left(n_{1}, \ldots, n_{k}\right) \in \mathbb{Z}_{+}^{k}$, each $n_{j} \neq 0$ and the radiant integral affine structure $\mathcal{A}_{n_{j}}$ on $B$ has linear holonomy generated by the matrix

$$
\left(\begin{array}{cc}
1 & 0 \\
-n_{j} & 1
\end{array}\right)
$$

Let $\mathfrak{l}_{Z_{\mathbf{n}}}$ be the linear holonomy of $\left(Z_{\mathbf{n}}, \mathcal{A}_{Z_{\mathbf{n}}}\right)$. All elements of the cohomology group

$$
\mathrm{H}^{2}\left(Z_{\mathbf{n}} ; \mathbb{Z}_{\mathfrak{l}_{Z_{\mathbf{n}}}^{-T}}^{2 k}\right)
$$

(which, by the above remark, is non trivial) can be realised as the Chern class of some regular Lagrangian bundle over $\left(Z_{\mathbf{n}}, \mathcal{A}_{Z_{\mathbf{n}}}\right)$. This example is interesting because each $\left(B, \mathcal{A}_{n_{j}}\right)$ is the affine model in the neighbourhood of a focus-focus singularity of a completely integrable Hamiltonian system, which is homeomorphic to a two torus pinched $n_{j}$ times, as shown in BF07, Zun97. Thus $\left(Z_{\mathbf{n}}, \mathcal{A}_{Z_{\mathbf{n}}}\right)$ is a local affine model for a product of focus-focus singularities. Such products occur naturally amongst non-degenerate singularities of Lagrangian fibrations, which have been classified topologically by Zung in [Zun96. However, it is not known whether there exist examples of non-degenerate singularities whose regular parts have non-trivial Chern class and, in particular, whether the Lagrangian bundles corresponding to non-zero cohomology classes in $\mathrm{H}^{2}\left(Z_{\mathbf{n}} ; \mathbb{Z}_{\mathfrak{r}_{Z_{\mathbf{n}}}^{-T}}^{2 k}\right)$ can be compactified to admit (non-degenerate) singularities.

\section{Conclusion}

This paper shows that the interplay between the symplectic geometry of Lagrangian bundles and affine differential geometry runs deep, allowing to use the methods of one subject to study the other and vice versa. The importance of the radiance obstruction in constructing Lagrangian fibrations has been recognised by other authors (e.g. Gross and Siebert in [GS06]) who use different techniques; in particular, the sheaf theoretic approach developed in GS06 yields an equivalent proof of the Main Result. In light of the examples of Section 5.3 and given the dearth of explicit examples of singularities of completely integrable Hamiltonian systems, the following is a natural question to ask.

Question. Are the examples of Section 5.3 the regular parts of some Lagrangian fibrations? If so, what can be said about the topology and symplectic geometry of the singularities?

Various recent works (e.g. [BVN10, GS06]) hint at the fact that there is a strong correlation between the affine geometry of the regular part of the base space of the fibration and the topology or symplectic geometry in a neighbourhood of a singularity. This is a subtle question, since many intervening factors, such as smoothness conditions, also play a crucial role. By studying integral affine manifolds with singularities (e.g. GS06), it may be possible to generalise the methods of this paper to either construct such examples explicitly or prove non-existence results, which would further clarify the nature of Lagrangian fibrations and of completely integrable Hamiltonian systems. 


\section{REFERENCES}

[AM55] L. Auslander and L. Markus. Holonomy of flat affinely connected manifolds. Annals of Math., 62:139 - 151, 1955.

[Arn78] V.I. Arnold. Mathematical Methods in Classical Mechanics. Springer-Verlag, 1978.

[Bai01] P. Baier. $\mathbb{T}^{3}$-fibrations on compact six-manifolds. arXiv:math/0109087v1, 2001.

[Bat88] L.M. Bates. Examples for obstructions to action-angle coordinates. Proc. Royal Soc. Edin, 110:27 - 30, 1988.

[Ben60] J.-P. Benzécri. Sur les variétés localement affines et localement projectives. Bull. Soc. Math. France, 88:229 - 332, 1960.

[BF07] L.M. Bates and F. Fassò. An affine model for the actions in an integrable system with monodromy. Regul. Chaotic Dyn., 12(6):675 - 679, 2007.

[BS92] L. Bates and J. Sniatycki. On action-angle variables. Arch. Rat. Mech. Anal., 120:337 - 343, 1992.

[BT99] R. Bott and L. W. Tu. Differential Forms in Algebraic Topology. Springer-Verlag, 1999.

[BVN10] A. Bolsinov and S. Vu Ngoc. Symplectic equivalence for integrable systems with common action integrals. in preparation, 2010.

[CB97] R.H. Cushman and L.M. Bates. Global Aspects of Classical Integrable Systems. Birkhauser, 1997.

[CV66] L.S. Charlap and A.T. Vasquez. The cohomology of group extensions. Trans. Amer. Math. Soc., 124(1):24-40, 1966.

[DD87] P. Dazord and P. Delzant. Le probleme general des variables actions-angles. J. Diff. Geom., 26:223 - 251, 1987.

[DK01] J.F. Davis and P. Kirk. Lecture Notes in Algebraic Topology. AMS, 2001.

[Dui80] J.J. Duistermaat. On global action-angle coordinates. Comm. Pure Appl. Math., 33:687 - 706, 1980.

[FGH81] D. Fried, W. M. Goldman, and M. W. Hirsch. Affine manifolds with nilpotent holonomy. Comment. Math. Helv., 56(4):487 - 523, 1981.

[FS07] F. Fassò and N. Sansonetto. Integrable almost-symplectic Hamiltonian systems. J. Math. Phys., 48(9), 2007.

[Gei92] H. Geiges. Symplectic structures on $\mathbb{T}^{2}$-bundles over $\mathbb{T}^{2}$. Duke Math Jour., 67(3):539 - 555, 1992.

[GH84] W.M. Goldman and M.W. Hirsch. The radiance obstruction and parallel forms on affine manifolds. Trans. Amer. Math. Soc., 286(2):629 - 649, 1984.

[GH86] W.M. Goldman and M.W. Hirsch. Affine manifolds and orbits of algebraic groups. Trans. Amer. Math. Soc., 295(1):175 $-198,1986$.

[Gro58] A. Grothendieck. A general theory of fibre spaces with structure sheaf. Technical report, University of Kansas, 1958.

[GS06] M. Gross and B. Siebert. Mirror symmetry via logarithmic degeneration data. I. J. Differential Geom., $72(2): 169-$ $338,2006$.

[Hus93] D. Husemoller. Fibre Bundles. Springer-Verlag, $3^{\text {rd }}$ rev. edition, 1993.

[Luk08] O. Lukina. Geometry of torus bundles in integrable Hamiltonian systems. PhD thesis, University of Groningen, 2008.

[McC85] J. McCleary. A User's Guide to Spectral Sequences. Publish or Perish, 1985.

[Mil58] J. Milnor. On the existence of a connection with curvature zero. Comment. Math. Helv., 32:215-223, 1958.

[Mis96] K.N. Mishachev. The classification of Lagrangian bundles over surfaces. Diff. Geom. Appl., 6:301 - 320, 1996.

[MM74] L. Markus and K. R. Meyer. Generic Hamiltonian dynamical systems are neither integrable nor ergodic. American Mathematical Society, Providence, R.I., 1974. Memoirs of the American Mathematical Society, No. 144.

[Ngo03] S. Vu Ngoc. On semi-global invariants for focus-focus singularities. Topology, 42(2):365 - 380, 2003.

[Sep10a] D. Sepe. Classification of Lagrangian fibrations over a Klein bottle. Geom. Dedicata, 149:347 - 362, 2010.

[Sep10b] D. Sepe. Topological classification of Lagrangian fibrations. Jour. Geom. Phy., 60:341 - 351, 2010.

[Sep11] D. Sepe. Almost Lagrangian obstruction. Differential Geom. Appl., 29(6):787-800, 2011.

[Sie67] J. Siegel. Higher order cohomology operations in local coefficient theory. Amer. J. Math., 89:909 - 931, 1967.

[Smi81] J. Smillie. An obstruction to the existence of affine structures. Inventiones Mathematicae, 64:411 - 415, 1981.

[SS11] N. Sansonetto and D. Sepe. Almost complex realisations of Poisson manifolds. in preparation, 2011.

[Vai94] I. Vaisman. Lectures on the geometry of Poisson manifolds, volume 118 of Progress in Mathematics. Birkhäuser Verlag, Basel, 1994.

[Wei71] A. Weinstein. Symplectic manifolds and their Lagrangian submanifolds. Advances in Math., 6:329 - $346,1971$.

[Whi78] G. W. Whitehead. Elements of homotopy theory. Springer-Verlag, 1978.

[Zun96] N.T. Zung. Symplectic topology of integrable Hamiltonian systems i: Arnold-Liouville with singularities. Compositio Math., 101:179 - 215, 1996.

[Zun97] N.T. Zung. A note on focus-focus singularities. Diff. Geom. and Appl., 7:123 - 130, 1997.

[Zun03] N.T. Zung. Symplectic topology of integrable Hamiltonian systems ii: Topological classification. Compositio Math., 138:125-156, 2003. 